\title{
Effects of Exogenous Hormones and Reproductive Factors on Female Melanoma: A Meta-Analysis
}

This article was published in the following Dove Press journal: Clinical Epidemiology

\author{
Qian Sun' \\ Hongyan Sun ${ }^{2}$ \\ Lele Cong' \\ Yang Zheng' \\ Nan Wu ${ }^{l}$ \\ Xianling Cong $\mathbb{D}^{\prime}$ \\ 'Department of Dermatology, China- \\ Japan Union Hospital of Jilin University, \\ Changchun, Jilin, People's Republic of \\ China; ${ }^{2}$ Tissue Bank, China-Japan Union \\ Hospital of Jilin University, Changchun, \\ jilin, People's Republic of China
}

\begin{abstract}
Epidemiological findings on the effects of hormones on melanoma risk have been inconsistent. We therefore conducted a meta-analysis to examine the relationship between exogenous hormonal and reproductive factors and the risk of melanoma in women. We performed a search of PubMed, Web of Science, and the China National Knowledge Infrastructure (CNKI) database through April 2020 for relevant studies. Based on heterogeneity, we performed the meta-analysis of the risk estimates using either fixed effect or random effect models. We identified 38 studies that met the analytical criteria, involving 3,571,910 participants. The results showed that long-term use of oral contraceptives (OC) may increase the risk of melanoma in women ( $\geq 5$ years [pooled $\mathrm{RR}=1.18$; 95\% CI: $1.07-$ $1.31 ; \mathrm{I}^{2}=0 \%$ ] and $\geq 10$ years [pooled $\left.\mathrm{RR}=1.25 ; 95 \% \mathrm{CI}: 1.06-1.48 ; \mathrm{I}^{2}=0 \%\right]$ ). Women who first used OC 15-19 years previously were more likely to develop melanoma (pooled $\mathrm{RR}=1.52 ; 95 \% \mathrm{CI}: 1.03-2.24 ; \mathrm{I}^{2}=0 \%$ ), while the years since the last use and the age at first use were not associated with the development of melanoma in women. Hormone replacement therapy (HRT) increased the incidence of melanoma in women (pooled $\mathrm{RR}=1.12$, 95\% CI: $1.02-1.24 ; \mathrm{I}^{2}=50 \%$ ) and was especially associated with an increased risk of superficial spreading melanoma (SSM) (pooled $\mathrm{RR}=1.26$; 95\% CI: 1.17-1.37; $\mathrm{I}^{2}=0 \%$ ), and estrogen and estradiol may be the main active agents that contribute to the increased risk of melanoma, but these results may be due to a combination of sun exposure factors. With regard to reproductive factors, decreased parity and being aged $\geq 20$ years at first birth may be associated with an increased risk of melanoma in females, while menopausal status and age at menarche are not associated with the incidence of melanoma in females. Further large-scale prospective studies are necessary to reveal new pathophysiological mechanisms and new therapeutic targets for cutaneous melanoma.
\end{abstract}

Keywords: oral contraceptives, hormone replacement therapy, menopause, menarche, parity

\section{Introduction}

In the early 2000 s, melanoma was still a potentially fatal malignancy. While the incidence of many tumor types has declined, the incidence of melanoma continues to increase. ${ }^{1}$ Several risk factors for melanoma have been identified, including exposure to ultraviolet light, fair skin and immunosuppression. ${ }^{2-5}$ In addition, the incidence trend showed that the incidence of melanoma in women remained higher than that in men until the age of 45 , after which the incidence of melanoma in men increased significantly but leveled off in women. ${ }^{4,6-8}$ Some studies reported cases of melanoma during pregnancy, pigmentation during oral contraceptives (OC) use, and blackening and enlargement of nevi during pregnancy. ${ }^{9-12}$ These descriptive findings raise the possibility that female sex hormones or reproductive effects may be involved in the etiology of female melanoma.
Correspondence: Xianling Cong

Department of Dermatology, China-Japan Union Hospital of Jilin University, Xiantai

Road I26, Changchun, Jilin I30033,

People's Republic of China

Tel +86-15904420533

Fax + 86-43I-89876626

Email congx|@jlu.edu.cn 
Since the 1970s, many epidemiological studies have focused on the possible relationship between female endocrine characteristics and melanoma, but there has been considerable debate about the potential impact of hormones on melanoma risk. The results of some previous studies have shown that women who have used OC or hormone replacement therapy (HRT) have a higher risk of developing melanoma than women who have never used OC or HRT, ${ }^{13-15}$ while other studies have found no additional risk, ${ }^{16-38}$ and two studies have even found a protective effect from OC and HRT use. ${ }^{39,40}$ In addition, two studies identified an increased risk of melanoma in women who have used OC for five years or more. ${ }^{13,32}$

Some studies suggest that a woman's reproductive history may affect her risk of melanoma, leading to speculation that the development of female melanoma may also be influenced by hormonal changes during pregnancy. However, this hypothesis has not been supported by all studies that have investigated the relationship between reproductive history and melanoma risk. Two studies have shown that women with multiple live births have a lower risk of melanoma than women with few or no live births $;{ }^{28,41}$ in contrast, one study found a positive correlation between the number of births and the risk of melanoma. ${ }^{31}$ One study also found that women who had their first child after age 30 had an increased risk of melanoma after adjusting for oral contraceptive use, ${ }^{32}$ while another study found that menarche before age 14 also significantly increased the risk of melanoma. ${ }^{24}$ Two studies identified an association between early birth and a reduced risk of melanoma. ${ }^{41,42}$ However, other studies have found no link between reproductive factors and melanoma risk. ${ }^{16,21,23,26,27,29,30,36,43}$

In this meta-analysis, we investigated the relationship between the incidence of cutaneous melanoma and the use of exogenous hormones (including OC and HRT) and reproductive factors (including parity, age of first child, age of menarche, and menopausal status).

\section{Materials and Methods}

This study was conducted in accordance with PRISMA guidelines. $^{44}$

\section{Data Sources and Search Strategy}

We performed a literature search to identify relevant articles available from PubMed, Web of Science and China National Knowledge Infrastructure (CNKI), from inception to April 2020, with no restrictions. We used the search terms "oral contraceptive", "hormonal replacement therapy", "menopausal hormone therapy", "estrogens", "parity", "menarche" or "menopause" in combination with "melanoma". Articles/titles/abstracts with these keywords were screened by two independent reviewers using preestablished inclusion criteria to identify relevant studies for inclusion in the systematic review. We additionally reviewed the reference lists of retrieved studies and recent reviews for potentially related studies.

\section{Inclusion and Exclusion Criteria}

Inclusion criteria were as follows: (1) published as original articles; (2) studies that evaluated and assessed the association between OC or HRT use or reproductive factors and the incidence melanoma; and (3) studies providing sufficient data to estimate the hazard ratio (HR), relative risk ratio (RR), or odds ratio (OR) and corresponding 95\% confidence intervals (CIs). The most recent and complete study was selected if studies from the same population were repeated.

The exclusion criteria were as follows: (1) studies that were published in reviews, case reports, editorial letters, comments, or responses; (2) studies that did not provide available data to estimate ORs, RRs, or HRs and 95\% CIs; and (3) studies that were repeated in the same study population.

\section{Data Extraction and Quality Assessment}

The data were independently extracted and cross-checked by two investigators. Any differences were settled by consensus.

The following information was extracted from each study: first author, publication year, type of study design, country, sample size or the number of cases and controls, confounders adjusted for, and adjusted or crude ORs, RRs or HRs (we presented all results as RR for simplicity) with 95\% CI for each exposure, including OC use, duration of use, years since last use, time since the first use, age at first use, HRT use, age at menarche, parity, age at first birth, and menopausal status.

The quality of each study was independently assessed by two researchers using the Newcastle-Ottawa Quality Assessment Scale (NOS). ${ }^{45}$ Scores ranged from 0 to 9 for quality assessment and studies with scores $\geq 6$ were rated as high quality.

\section{Statistical Analysis}

Adjusted or crude measures of effect were extracted from the included studies. Where adjusted measures were 
available, these were preferred. Since melanoma is a rare disease, ORs were considered an approximation of RR. ${ }^{46}$ All included studies evaluating HRs adopted the COX model; therefore, HRs were directly considered as RRs. The pooled analysis of all reports was performed using the Inverse Variance method. Forest plots were drawn to summarize the information from individual studies and the pooled effect size of the study objects. Sensitivity analysis was performed by omitting one study at a time to evaluate the potential bias and robustness of the overall risk estimate. Heterogeneity between studies was determined by Chi-square test, Cochran's Q test, and $\mathrm{I}^{2}$ statistics. ${ }^{47} \mathrm{I}^{2}$ values $\leq 25 \%$ indicated no heterogeneity, $\leq 50 \%$ indicated minimal heterogeneity, $\leq 75 \%$ indicated moderate heterogeneity, and $>75 \%$ indicated significant heterogeneity. Statistical methods for meta-analysis included the fixed effect model and the random effect model. The analysis model was selected according to the heterogeneity. The random effects model was used to account for variation within and between studies. ${ }^{47}$ P-values $<0.10$ and/or $\mathrm{I}^{2}$ $>50 \%$ indicated the presence of heterogeneity and the random-effects model (DerSimonian-Laird method) was applied to partially eliminate the effects of heterogeneity. Otherwise, the fixed-effects model (Mante-Haenszel method) was used. ${ }^{48}$ We performed subgroup analyses of the groups with a high number of studies based on study design type, geographical area, whether confounders were corrected for, whether education was corrected for, and whether daylight exposure was corrected for to assess the impact of these variables on the results. Nested case-control studies were classified as case-control studies for the purpose of analysis. Potential publication bias was assessed by both the Begg rank correlation test and the Egger linear regression test. ${ }^{49,50}$

All analyses were performed using Review Manager version 5.3 (The Cochrane Collaboration, Copenhagen: The Nordic Cochrane Centre, 2012) and STATA version 12.0 (STATA Corporation, College Station, TX, USA), with significance defined as a P-value less than 0.05 .

\section{Results}

\section{Study Characteristics}

Using the search strategy described above, 2461 citations were initially retrieved. After screening the titles, abstracts, publication types, and full texts of each article, 66 articles were identified that investigated the correlation between the use of OC or HRT or reproductive factors and the incidence of female melanoma. Of these studies, 28 were excluded, including 6 for which no data were available, 21 because they were case reports, reviews, or letters, and one because it was a study of a repeating population. A final total of 38 articles were included in the meta-analysis (Figure 1). ${ }^{13-43,51,57}$ Among these, 11 studies only evaluated the relationship between OC use and the incidence of melanoma in females, ${ }^{13,17-19,22,25,35,39,52,53,57} 4$ studies only investigated HRT, ${ }^{15,33,37,56} 4$ assessed both OC and HRT, ${ }^{14,20,38,40} 5$ investigated OC and reproductive factors, ${ }^{21,23,24,26,51}$ and 9 assessed OC, HRT and reproductive factors. ${ }^{16,27-32,34,36}$ A further 5 studies only investigated the influence of reproductive factors on the incidence of female melanoma. ${ }^{41-43,54,55}$ A total of 3,571,910 participants from the United States, Britain, Poland, Australia, Canada, Denmark, Sweden, Italy, France, Russia, Netherlands, Norway, Finland, Spain, Germany, Greece, and the United Kingdom were enrolled, with patients ranging from 107 to $1,725,627$ in each study. There were 20 case-control studies, ${ }^{14-16,18,20-28,30-32,34,39-41} 14$ cohort studies, ${ }^{13,29,33,35-38,42,43,52,53,55-57} 3$ nested case-control studies, ${ }^{19,51,54}$ and 1 study that included both cohort and case-control studies. ${ }^{17}$ Details of the 38 studies included in the analysis are shown in Table 1.

The quality of each study included in our meta-analysis was evaluated according to the NOS. The highest scores for case-control and cohort studies were 8 and 9, respectively. Quality scores of the studies ranged from 5 to 9 , with an average of 7.2 (Table 1). All of the studies were considered adequate for inclusion in this meta-analysis.

\section{Pooled Analyses \\ The Use of OC}

Twenty-nine articles assessed the risk of melanoma in women who used OC, ${ }^{13,14,16-32,34-36,38-40,51,53,57}$ but two of these were not included in the analysis because the study population was repeated. ${ }^{34,35}$ Of a total of 18 case-control studies $^{14,16-18,20,28,30-32,39,40}$ and 2 nested case-control studies, ${ }^{19,51} 1$ study showed a significantly increased risk of melanoma associated with OC use, ${ }^{14}$ and 3 showed a reduced risk. ${ }^{26,39,40}$ Of a total of 7 cohort studies, ${ }^{13,29,36,38,52,53,57}$ only 1 showed a significantly increased risk. ${ }^{38}$ Among all case-control studies, the combined melanoma risk for OC use was 0.98 (95\% CI: 0.83 1.15; $P=0.77)$. The combined risk based on all cohort studies was 1.06 (95\% CI: $0.98-1.14 ; P=0.17)$, while the combined pooled risk was 0.99 (95\% CI: $0.90-1.10$; $P=0.87)$, with moderate heterogeneity $\left(\mathrm{I}^{2}=54 \%\right)$ (Table 2 , 


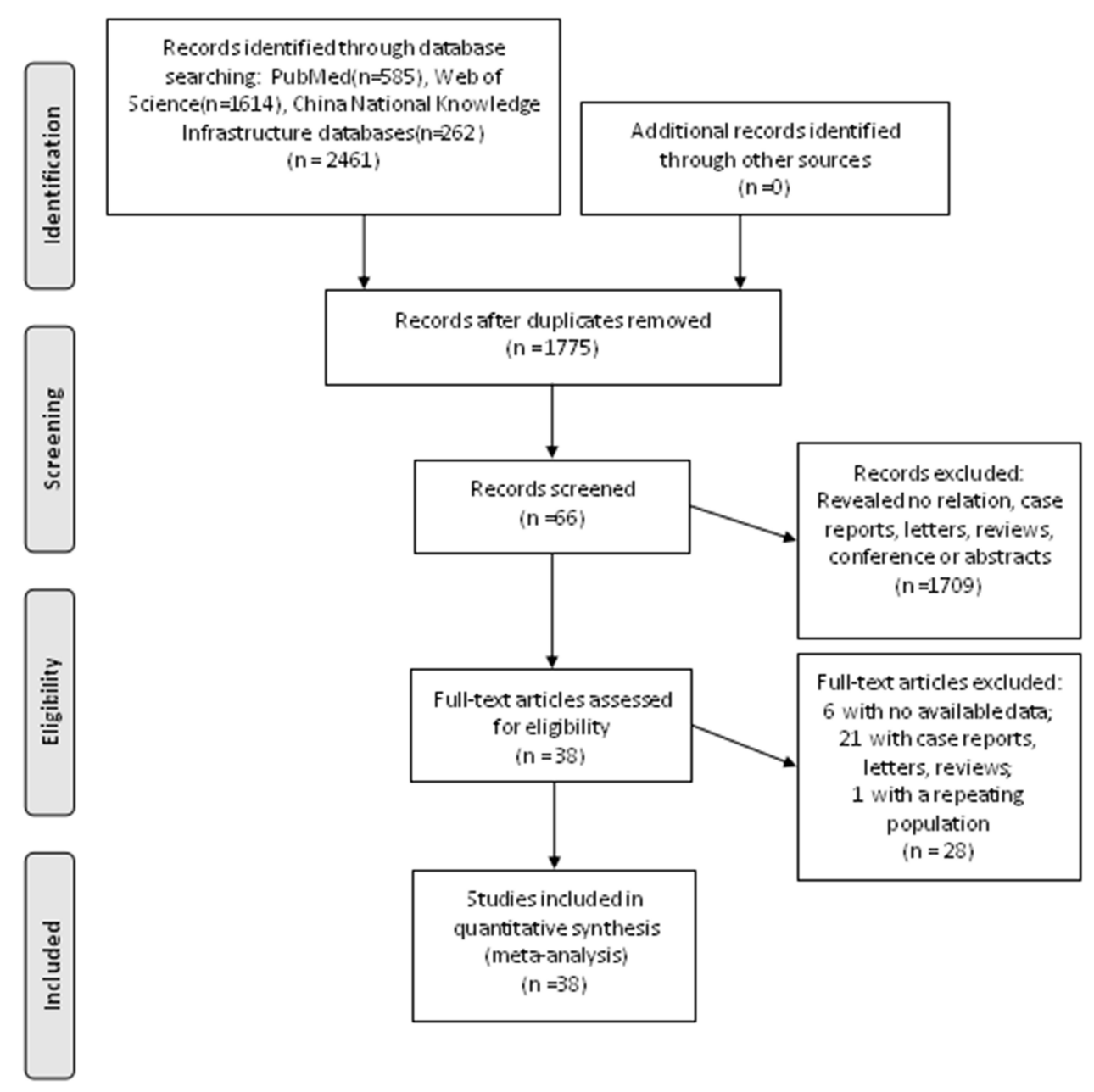

Figure I Flowchart of study selection process.

Figure 2). This suggests that the use of OC is not related to the development of female melanoma.

Six articles evaluated the relationship between the use of OC and the incidence of different histological subtypes of female melanoma. ${ }^{20,21,23,26,32,35}$ As shown in Table 2, all 6 of these manuscripts evaluated the relationship between OC use and the incidence of superficial spreading melanoma (SSM) in women, and 4 of them also assessed the risk of nodular melanoma (NM) in women using OC..$^{20,21,26,35}$ Women using OC had a relative risk of 1.01 (95\% CI: $\left.0.87-1.18 ; \mathrm{I}^{2}=27 \%\right)$ for SSM and 0.84 (95\% CI: $0.55-1.30 ; \mathrm{I}^{2}=23 \%$ ) for NM (Supplementary figure 1). This suggests that the use of OC is independent of the occurrence of SSM and NM in women.

Twenty-four articles evaluated the relationship between the duration of $\mathrm{OC}$ use and the incidence of melanoma in women. ${ }^{13,14,16-29,32,34,36,38,51-53}$ Seven of these were not included in the analysis due to different classification methods from other literature or lack of available data, ${ }^{14,17,23,28,36,52,53}$ and one article was not included in the analysis because the study population was repeated (Table 1) ${ }^{34}$ As shown in Table 2, there was no statistically significant risk of melanoma in women with a duration of use of $<2$ years (pooled RR: 0.87 ; 95\% CI: $0.66-1.14$; $\mathrm{I}^{2}=0 \%$ ) or $<5$ years (pooled RR: 0.96 ; 95\% CI: $0.82-1.13$; $\mathrm{I}^{2}=59 \%$ ) (Figure $3 \mathrm{~A}$ and $\mathrm{B}$ ). A duration of use $\geq 5$ years (pooled RR: 1.18; 95\% CI: 1.07-1.31; $P=0.001$ ) and $\geq 10$ years (pooled RR: 1.25 ; 95\% CI: $1.06-1.48, P=0.008$ ) showed significant risks without heterogeneity $\left(\mathrm{I}^{2}=0 \%\right.$ for both) (Figure 3C and D). This suggests that longterm use of OC may increase the risk of melanoma in women.

Given that an incubation period of several years is required to increase the risk of melanoma, we also analyzed the impact of time since first use, years since last use, and age at first use on the risk of female melanoma. Four articles evaluated the relationship between time since the first use and incidence of female melanoma, ${ }^{13,24-26}$ but 
Table I The Detailed Characteristics of the Included Studies

\begin{tabular}{|c|c|c|c|c|c|c|c|}
\hline $\begin{array}{l}\text { First } \\
\text { Author, } \\
\text { Publication } \\
\text { Year }\end{array}$ & $\begin{array}{l}\text { Study } \\
\text { Design }\end{array}$ & Country & $\begin{array}{l}\text { Sample Size } \\
\text { or Cases/ } \\
\text { Controls }\end{array}$ & Age & Risk Factors Included & Confounders Adjusted & $\begin{array}{l}\text { NOS } \\
\text { Score }\end{array}$ \\
\hline \multirow[t]{2}{*}{ Beral, $1977^{17}$} & Cohort & US & 17,942 & $17-59$ & $\begin{array}{l}\text { OC use*, Duration of } \\
\text { OC use* }\end{array}$ & No & \multirow[t]{2}{*}{7} \\
\hline & $\begin{array}{l}\text { Case- } \\
\text { control }\end{array}$ & & $37 / 74$ & $20-59$ & OC use & No & \\
\hline $\begin{array}{l}\text { Adam, 198I } \\
18\end{array}$ & $\begin{array}{l}\text { Case- } \\
\text { control }\end{array}$ & Britain & $169 / 507$ & $15-49$ & $\begin{array}{l}\text { OC use, duration of } O C \\
\text { use }\end{array}$ & No & 7 \\
\hline Bain, $1982^{19}$ & $\begin{array}{l}\text { Nested } \\
\text { case- } \\
\text { control }\end{array}$ & US & $|4| / 2820$ & $30-55$ & $\begin{array}{l}\text { OC use, duration of } O C \\
\text { use }\end{array}$ & $\begin{array}{l}\text { Age at diagnosis, state of } \\
\text { residence, parity, age at first } \\
\text { pregnancy, height, and prior hair } \\
\text { dye use }\end{array}$ & 8 \\
\hline Holly, $1983^{32}$ & $\begin{array}{l}\text { Case- } \\
\text { control }\end{array}$ & US & $87 / 863$ & $37-74$ & $\begin{array}{l}\text { OC use } \mathrm{m}^{\mathrm{m}} \text {, duration of } \\
\text { OC use, HRT use, age at } \\
\text { first birth*, parity }\end{array}$ & Age & 8 \\
\hline Beral, $1984^{20}$ & $\begin{array}{l}\text { Case- } \\
\text { control }\end{array}$ & Australia & $287 / 574$ & $15-24$ & $\begin{array}{l}\text { OC use }{ }^{m} \text {, duration of } \\
O C \text { use, HRT use }\end{array}$ & No & 6 \\
\hline $\begin{array}{l}\text { Holman, } \\
1984^{21}\end{array}$ & $\begin{array}{l}\text { Case- } \\
\text { control }\end{array}$ & Australia & $276 / 276$ & $\begin{array}{l}\text { Mean } 44.9 \\
(10-79)\end{array}$ & $\begin{array}{l}\text { OC use }{ }^{\mathrm{m}} \text {, duration of } \\
\text { OC use, years since last } \\
\text { use, age at menarche, } \\
\text { parity }\end{array}$ & No & 7 \\
\hline $\begin{array}{l}\text { Helmrich, } \\
1984^{22}\end{array}$ & $\begin{array}{l}\text { Case- } \\
\text { control }\end{array}$ & US, Canada & $160 / 160$ & $20-59$ & $\begin{array}{l}\text { OC use, duration of } O C \\
\text { use }\end{array}$ & Age & 6 \\
\hline $\begin{array}{l}\text { Osterlind, } \\
1988^{16}\end{array}$ & $\begin{array}{l}\text { Case- } \\
\text { control }\end{array}$ & Denmark & $280 / 536$ & $20-79$ & $\begin{array}{l}\text { OC } \text { use }^{\mathrm{a}} \text {, duration of } O C \\
\text { use }^{\mathrm{a}}, \mathrm{HRT} \text { use } \mathrm{b}^{\mathrm{b}} \text {, age at } \\
\text { menarche }^{\mathrm{a}} \text {, parity } \\
\text { menopausal status }^{\mathrm{a}}\end{array}$ & $\begin{array}{l}\text { aAge at diagnosis, naevi, freckles, } \\
\text { hair color and sunbathing; }{ }^{\text {bage at }} \\
\text { diagnosis, naevi and sunbathing; } \\
\text { cno }\end{array}$ & 8 \\
\hline $\begin{array}{l}\text { Adami, } 1989 \\
33\end{array}$ & Cohort & Sweden & 23,244 & Mean 54.5 & HRT use & No & 7 \\
\hline $\begin{array}{l}\text { Zanetti, } 1990 \\
23\end{array}$ & $\begin{array}{l}\text { Case- } \\
\text { control }\end{array}$ & Italy & $186 / 205$ & $19-92$ & $\begin{array}{l}\text { OC use }{ }^{m} \text {, duration of } \\
\text { OC use*, parity, age at } \\
\text { first birth }\end{array}$ & $\begin{array}{l}\text { Age, education, skin reaction to } \\
\text { sun exposure, sunburns in } \\
\text { childhood, and weeks of holiday } \\
\text { on the beach }\end{array}$ & 8 \\
\hline Lê, $1992^{24}$ & $\begin{array}{l}\text { Case- } \\
\text { control }\end{array}$ & France & $91 / 149$ & $\begin{array}{l}\text { Mean } 34.2 \\
(S D=7.6) \\
\text { for cases; } \\
34.1 \text { (SD = } \\
7.4 \text { ) for } \\
\text { controls }\end{array}$ & $\begin{array}{l}\text { OC } \text { use }^{\mathrm{a}} \text {, duration of } O C \\
\text { use }^{\mathrm{b}} \text {, time since the first } \\
\text { use }^{\mathrm{b}} \text {, age at first use }{ }^{\mathrm{b}} \text {, age } \\
\text { at menarche }{ }^{* \mathrm{~b}} \text {, parity }\end{array}$ & $\begin{array}{l}{ }^{a} \mathrm{No} \text {; }{ }^{b} \text { age at menarche, color of } \\
\text { hair, color of eyes, skin } \\
\text { complexion, types of skin, total } \\
\text { sunlight exposure }\end{array}$ & 7 \\
\hline $\begin{array}{l}\text { Palmer, } 1992 \\
25\end{array}$ & $\begin{array}{l}\text { Case- } \\
\text { control }\end{array}$ & US & $615 / 2107$ & $\begin{array}{l}\text { Median } 40 \\
(18-64)\end{array}$ & $\begin{array}{l}\text { OC } \text { use }^{a} \text {, duration of } O C \\
\text { use }^{a} \text {, years since last } \\
\text { use }^{b} \text {, time since the first } \\
\text { use }^{b} \text {, age at first use }\end{array}$ & $\begin{array}{l}\text { aNo; age, geographic region, year } \\
\text { of interview, years of education, } \\
\text { religion, body mass index, } \\
\text { menopausal status, and skin type }\end{array}$ & 7 \\
\hline
\end{tabular}

(Continued) 
Table I (Continued).

\begin{tabular}{|c|c|c|c|c|c|c|c|}
\hline $\begin{array}{l}\text { First } \\
\text { Author, } \\
\text { Publication } \\
\text { Year }\end{array}$ & $\begin{array}{l}\text { Study } \\
\text { Design }\end{array}$ & Country & $\begin{array}{l}\text { Sample Size } \\
\text { or Cases/ } \\
\text { Controls }\end{array}$ & Age & Risk Factors Included & Confounders Adjusted & $\begin{array}{l}\text { NOS } \\
\text { Score }\end{array}$ \\
\hline $\begin{array}{l}\text { Zaridze, } 1992 \\
39\end{array}$ & $\begin{array}{l}\text { Case- } \\
\text { control }\end{array}$ & Russia & $96 / 96$ & NA & $O C$ use & $\begin{array}{l}\text { Skin color, freckles on arms, } \\
\text { raised naevi on arms, naevi on } \\
\text { trunk diameter, sunbathing at age } \\
\text { I8-20 }\end{array}$ & 6 \\
\hline Holly, $1994^{34}$ & $\begin{array}{l}\text { Case- } \\
\text { control }\end{array}$ & US & $452 / 930$ & $25-59$ & $\begin{array}{l}\text { OC use }{ }^{\# a} \text {, duration of } \\
\text { OC use }{ }^{\# a}, H R T \text { use }{ }^{a} \text {, age } \\
\text { at menarche*b, } \\
\text { menopausal status }\end{array}$ & ${ }^{\mathrm{a}} \mathrm{Age}$ and education; ${ }^{\mathrm{b}}$ age & 8 \\
\hline Holly, $1995^{26}$ & $\begin{array}{l}\text { Case- } \\
\text { control }\end{array}$ & US & $452 / 930$ & $25-59$ & $\begin{array}{l}\text { OC } \text { use }^{\text {ma }} \text {, duration of } \\
\text { OC use } \text {, years since last } \\
\text { use }^{\text {b }} \text {, time since the first } \\
\text { use }^{* b} \text {, parity }{ }^{b} \text { age at first } \\
\text { birth }^{* b}\end{array}$ & ${ }^{a}$ No, bage & 8 \\
\hline $\begin{array}{l}\text { Lambe, } 1996 \\
41\end{array}$ & $\begin{array}{l}\text { Case- } \\
\text { control }\end{array}$ & Sweden & $4779 / 23,888$ & $24-65$ & Parity, age at first birth & Age at first birth and parity & 7 \\
\hline $\begin{array}{l}\text { Westerdahl, } \\
1996^{28}\end{array}$ & $\begin{array}{l}\text { Case- } \\
\text { control }\end{array}$ & Sweden & $400 / 640$ & $15-75$ & $\begin{array}{l}\text { OC use }{ }^{a} \text {, duration of } O C \\
\text { use } e^{* a}, H^{2} \text { use }^{a}, \text { parity }^{b}, \\
\text { age at first birth }\end{array}$ & $\begin{array}{l}{ }^{a} \text { Host factors (ie hair color and } \\
\text { raised naevi) and history of } \\
\text { sunburns; bhost factors, history } \\
\text { of sunburn and age at first birth; } \\
\text { chost factors, history of sunburns } \\
\text { and number of live births }\end{array}$ & 8 \\
\hline $\begin{array}{l}\text { Smith, } 1998 \\
27\end{array}$ & $\begin{array}{l}\text { Case- } \\
\text { control }\end{array}$ & US & $308 / 233$ & Mean 53.5 & $\begin{array}{l}\text { OC } \text { use }^{a} \text {, duration of } O C \\
\text { use }^{a} \text {, age at first OC } \\
\text { use }^{* a} \text {, HRT use }{ }^{a} \text {, parity } \\
\text { menopausal status }^{c}\end{array}$ & $\begin{array}{l}\text { aAge, marital status, hair color, } \\
\text { number of arm nevi and sun } \\
\text { exposure Index; }{ }^{\text {bage, marital }} \\
\text { status, socloeconomic status, } \\
\text { freckling, hair color, number of } \\
\text { arm naevi, skin color, sun } \\
\text { exposure index and age at } \\
\text { menopause; cage, marital status, } \\
\text { freckling, hair color, number of } \\
\text { arm naevi, history of severe } \\
\text { sunburn, sun exposure index, } \\
\text { number of pregnancies lasting }>6 \\
\text { months. }\end{array}$ & 8 \\
\hline $\begin{array}{l}\text { Feskanich, } \\
1999^{13}\end{array}$ & Cohort & US & $\begin{array}{l}\text { I83,693 } \\
\text { (NHS:79,57I; } \\
\text { NHS II:104,I22) }\end{array}$ & $30-55$ & $\begin{array}{l}\text { OC use, duration of } O C \\
\text { use, years since last use, } \\
\text { time since the first use, } \\
\text { age at first use }\end{array}$ & $\begin{array}{l}\text { Age, follow-up cycle, skin } \\
\text { reaction after } 2 \text { hours of sun } \\
\text { exposure during childhood, } \\
\text { number of sunburns over lifetime } \\
\text { (NHS) or during teenage years } \\
\text { (NHS II), number of moles on } \\
\text { left arm (NHS) or on lower legs } \\
\text { (NHS II), hair color, family } \\
\text { history of melanoma, parity, } \\
\text { height, and body mass index }\end{array}$ & 7 \\
\hline
\end{tabular}

(Continued) 
Table I (Continued).

\begin{tabular}{|c|c|c|c|c|c|c|c|}
\hline $\begin{array}{l}\text { First } \\
\text { Author, } \\
\text { Publication } \\
\text { Year }\end{array}$ & $\begin{array}{l}\text { Study } \\
\text { Design }\end{array}$ & Country & $\begin{array}{l}\text { Sample Size } \\
\text { or Cases/ } \\
\text { Controls }\end{array}$ & Age & Risk Factors Included & Confounders Adjusted & $\begin{array}{l}\text { NOS } \\
\text { Score }\end{array}$ \\
\hline $\begin{array}{l}\text { Young, 200I } \\
51\end{array}$ & $\begin{array}{l}\text { Nested } \\
\text { case- } \\
\text { control }\end{array}$ & Australia & $14 / 93$ & $19-46$ & $\begin{array}{l}\text { OC use, duration of } O C \\
\text { use, age at menarche, } \\
\text { parity, age at first birth* }\end{array}$ & Cohort entry year and entry age & 6 \\
\hline $\begin{array}{l}\text { Freedman, } \\
2003^{29}\end{array}$ & Cohort & US & 68,588 & NA & $\begin{array}{l}\text { OC use, duration of OC } \\
\text { use, age at first use*, } \\
\text { HRT use, age at } \\
\text { menarche*, parity, age at } \\
\text { first birth, menopausal } \\
\text { status }\end{array}$ & $\begin{array}{l}\text { Gender, alcohol intake, years } \\
\text { smoked, skin pigmentation, hair } \\
\text { color, personal history of non- } \\
\text { melanoma skin cancer, decade } \\
\text { began work as a technologist, } \\
\text { education, and proxy measures } \\
\text { for residential childhood and } \\
\text { adult sunlight exposure }\end{array}$ & 6 \\
\hline $\begin{array}{l}\text { Naldi, } 2005 \\
30\end{array}$ & $\begin{array}{l}\text { Case- } \\
\text { control }\end{array}$ & Italy & $316 / 308$ & NA & $\begin{array}{l}\text { OC use, HRT use, parity, } \\
\text { age at first birth*, } \\
\text { menopausal status }\end{array}$ & $\begin{array}{l}\text { Age, education, body mass index, } \\
\text { number of melanocytic nevi, } \\
\text { pigmentary traits, history of } \\
\text { sunburns, and reaction to sun } \\
\text { exposure }\end{array}$ & 7 \\
\hline $\begin{array}{l}\text { Neale, } 2005 \\
42\end{array}$ & Cohort & Sweden & $1,234,967$ & NA & Parity*, age at first birth & $\begin{array}{l}\text { Twinning, number of maternities, } \\
\text { age at first birth and date of birth } \\
\text { of the mother }\end{array}$ & 7 \\
\hline $\begin{array}{l}\text { Vessey, } 2006 \\
52\end{array}$ & Cohort & UK & 17,032 & $25-39$ & $\begin{array}{l}\text { OC use, duration of } O C \\
\text { use*, years since last use* }\end{array}$ & Age, social class, smoking, height & 7 \\
\hline $\begin{array}{l}\text { Hannaford, } \\
2007^{53}\end{array}$ & Cohort & UK & $\begin{array}{l}91,893 \text { (Main } \\
\text { dataset: } 45,923 \text {; } \\
\text { General } \\
\text { practitioner } \\
\text { observation } \\
\text { dataset:45,970) }\end{array}$ & $\begin{array}{l}\text { Mean } 29 \\
\pm 6.6\end{array}$ & $\begin{array}{l}\text { OC use, duration of OC } \\
\text { use*, years since last use }\end{array}$ & $\begin{array}{l}\text { Main dataset: age, parity, } \\
\text { smoking, and social status; } \\
\text { General practitioner observation } \\
\text { dataset: age, parity, smoking, } \\
\text { social status, and ever use of } \\
\text { hormone replacement therapy }\end{array}$ & 7 \\
\hline Lea, $2007^{31}$ & $\begin{array}{l}\text { Case- } \\
\text { control }\end{array}$ & US & $318 / 395$ & $20-79$ & $\begin{array}{l}\text { OC use }{ }^{a}, H R T \text { use } \\
\text { parity }^{\mathrm{b}}\end{array}$ & $\begin{array}{l}\text { a'Age group, education, study } \\
\text { location, dysplastic nevus status, } \\
\text { total number of nevi, extent of } \\
\text { freckling, and never or ever } \\
\text { pregnant; bage group ( } 20-29 \text {, } \\
30-39,40 \text { years), education, } \\
\text { study location, dysplastic nevus } \\
\text { status, total number of nevi, } \\
\text { extent of freckling, and } \\
\text { exogenous hormone use of any } \\
\text { kind }\end{array}$ & 7 \\
\hline $\begin{array}{l}\text { Hannibal, } \\
2008^{54}\end{array}$ & $\begin{array}{l}\text { Nested } \\
\text { case- } \\
\text { control }\end{array}$ & Denmark & 54,362 & NA & Parity, age at first birth* & $\begin{array}{l}\text { Age at cohort entry (18-26; } \\
\text { 27-30; 31-36; 37-55) and } \\
\text { calendar year of cohort entry } \\
\text { (1963-1977; 1978-1984; } \\
\text { 1985-1989; 1990-1996; } \\
\text { 1997-1998) }\end{array}$ & 7 \\
\hline
\end{tabular}

(Continued) 
Table I (Continued).

\begin{tabular}{|c|c|c|c|c|c|c|c|}
\hline $\begin{array}{l}\text { First } \\
\text { Author, } \\
\text { Publication } \\
\text { Year }\end{array}$ & $\begin{array}{l}\text { Study } \\
\text { Design }\end{array}$ & Country & $\begin{array}{l}\text { Sample Size } \\
\text { or Cases/ } \\
\text { Controls }\end{array}$ & Age & Risk Factors Included & Confounders Adjusted & $\begin{array}{l}\text { Nos } \\
\text { Score }\end{array}$ \\
\hline $\begin{array}{l}\text { Koomen, } \\
2009^{14}\end{array}$ & $\begin{array}{l}\text { Case- } \\
\text { control }\end{array}$ & Netherlands & $778 / 4072$ & $\begin{array}{l}\text { Case: mean } \\
53.6 \\
\text { Control: } \\
54.6\end{array}$ & $\begin{array}{l}\text { OC use, duration of OC } \\
\text { use*, HRT use }\end{array}$ & $\begin{array}{l}\text { The total number of unique } \\
\text { prescriptions dispensed } \\
\text { (excluding estrogens) and the } \\
\text { use of nonsteroidal anti- } \\
\text { inflammatory drugs }\end{array}$ & 7 \\
\hline $\begin{array}{l}\text { Kvaskoff, } \\
2011^{55}\end{array}$ & Cohort & France & 91,972 & $40-65$ & $\begin{array}{l}\text { Age at menarche, parity*, } \\
\text { age at first birth, } \\
\text { menopausal status }\end{array}$ & $\begin{array}{l}\text { Age, hair color, skin complexion, } \\
\text { number of nevi, number of } \\
\text { freckles, skin sensitivity to sun } \\
\text { exposure, mean ultraviolet } \\
\text { radiation dose in regions of birth } \\
\text { and of residence at baseline, and } \\
\text { educational level }\end{array}$ & 8 \\
\hline $\begin{array}{l}\text { De Giorgi, } \\
2017^{40}\end{array}$ & $\begin{array}{l}\text { Case- } \\
\text { control }\end{array}$ & Italy & $605 / 592$ & Median 50 & OC use, HRT use & No & 5 \\
\hline $\begin{array}{l}\text { Botteri, } 2017 \\
56\end{array}$ & Cohort & Norway & 684,696 & $45-79$ & HRT use & $\begin{array}{l}\text { Age, number of children, age at } \\
\text { first birth, education, marital } \\
\text { status, sun exposure, use of } \\
\text { antihypertensives, antidiabetics, } \\
\text { statins and thyroid therapy }\end{array}$ & 7 \\
\hline $\begin{array}{l}\text { Mueller, } 2018 \\
57\end{array}$ & Cohort & US & 77,293 & $18-40$ & OC use & No & 5 \\
\hline $\begin{array}{l}\text { Cervenka, } \\
2018^{35}\end{array}$ & Cohort & France & 79,365 & $40-65$ & $\begin{array}{l}O C \text { use }^{\# m} \text {, duration of } \\
\text { OC use, years since last } \\
\text { use*, age at first use }^{\#}\end{array}$ & $\begin{array}{l}\text { Age, stratified according to year } \\
\text { of birth, residential UV exposure } \\
\text { at birth and at inclusion, } \\
\text { pigmentary traits and family } \\
\text { history of skin cancer. }\end{array}$ & 8 \\
\hline $\begin{array}{l}\text { Donley, } 2019 \\
36\end{array}$ & Cohort & US & 167,503 & $50-71$ & $\begin{array}{l}\text { OC use, duration of OC } \\
\text { use*, HRT use, age at } \\
\text { menarche*, parity*, age } \\
\text { at first birth }\end{array}$ & $\begin{array}{l}\text { Age, ambient ultraviolet } \\
\text { radiation quartile, education, } \\
\text { high school, some college, } \\
\text { college or graduate school, } \\
\text { body mass index, smoking } \\
\text { status, marriage, family history } \\
\text { of cancer, colonoscopy or } \\
\text { sigmoidoscopy, menopausal } \\
\text { hormone therapy }\end{array}$ & 8 \\
\hline $\begin{array}{l}\text { Hicks, } 2019 \\
15\end{array}$ & $\begin{array}{l}\text { Case- } \\
\text { control }\end{array}$ & Denmark & $8279 / 165,580$ & $45-85$ & HRT use & Age and calendar time & 7 \\
\hline
\end{tabular}

(Continued) 
Table I (Continued).

\begin{tabular}{|c|c|c|c|c|c|c|c|}
\hline $\begin{array}{l}\text { First } \\
\text { Author, } \\
\text { Publication } \\
\text { Year }\end{array}$ & $\begin{array}{l}\text { Study } \\
\text { Design }\end{array}$ & Country & $\begin{array}{l}\text { Sample Size } \\
\text { or Cases/ } \\
\text { Controls }\end{array}$ & Age & Risk Factors Included & Confounders Adjusted & $\begin{array}{l}\text { NOS } \\
\text { Score }\end{array}$ \\
\hline $\begin{array}{l}\text { Støer, } 2019 \\
43\end{array}$ & Cohort & Norway & $165,7 \mid 2$ & Mean 49 & $\begin{array}{l}\text { Age at menarche*, parity }{ }^{\mathrm{a}} \text {, } \\
\text { age at first birth*, } \\
\text { menopausal status }^{\mathrm{b}}\end{array}$ & 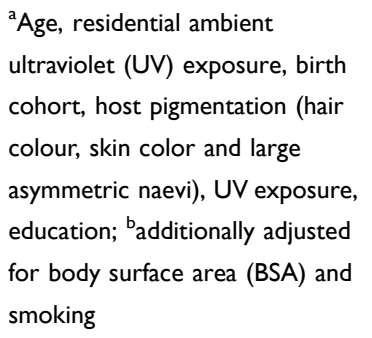 & 8 \\
\hline $\begin{array}{l}\text { Cervenka, } \\
2019^{37}\end{array}$ & Cohort & France & 75,523 & $40-65$ & HRT use ${ }^{\#}$ & $\begin{array}{l}\text { Age, stratified according to year } \\
\text { of birth and adjusted for } \\
\text { residential UV exposure at birth } \\
\text { and at inclusion, pigmentary } \\
\text { traits, and family history of skin } \\
\text { cancer }\end{array}$ & 9 \\
\hline $\begin{array}{l}\text { Cervenka, } \\
2020^{38}\end{array}$ & Cohort & $\begin{array}{l}\text { France, Italy, } \\
\text { Spain, UK, } \\
\text { Netherlands, } \\
\text { Germany, } \\
\text { Denmark, } \\
\text { Norway, Greece, } \\
\text { Sweden }\end{array}$ & $\begin{array}{l}\text { 469,24I(OC: } \\
\text { 334,483; HRT: } \\
\text { I34,758) }\end{array}$ & $\begin{array}{l}\text { Mean 5I.I } \\
\text { (SD9.7) }\end{array}$ & $\begin{array}{l}\text { OC use, duration of OC } \\
\text { use, age at first OC use*, } \\
\text { HRT use }\end{array}$ & $\begin{array}{l}\text { Center, age at recruitment, } \\
\text { education, age at menarche, } \\
\text { length of menstrual cycles, } \\
\text { number of full term pregnancies, } \\
\text { menopausal status, height, body } \\
\text { mass index and tobacco use }\end{array}$ & 8 \\
\hline
\end{tabular}

Notes: *The data were not included in the summary analysis due to different classification methods from other literature or lack of available data. ${ }^{\#}$ The data were not

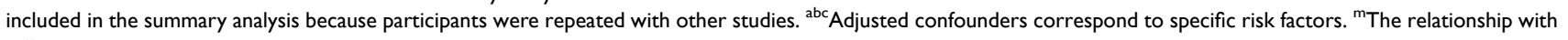
different pathological types was evaluated.

Abbreviations: OC, oral contraceptives; HRT, hormone replacement therapy; NA, not available; NHS, the Nurses' Health Study; NOS, the Newcastle-Ottawa Quality Assessment Scale; UV, ultraviolet.

one of these was not included in the analysis due to different classification methods from the other literature. ${ }^{26}$ There was an increased risk of melanoma in women for whom 15-19 years had elapsed since the first use (pooled RR: 1.52 ; 95\% CI: $1.03-2.24 ; P=0.03$ ), while there was no statistically significant risk of melanoma in women for whom < 15 years (pooled RR: 0.96; 95\% CI: 0.73-1.26; $P=0.75$ ) or $\geq 20$ years (pooled RR: 1.14 ; 95\% CI: $0.81-$ $1.61 ; P=0.44$ ) had elapsed since the first use. There was no heterogeneity for any of these $\left(I^{2}=0 \%\right.$ for all $)$ (Supplementary figure 2).

Seven articles assessed the relationship between elapsed time since last use and incidence of melanoma in women. $^{13,21,25,26,35,52,53}$ Two of these studies were not included in the analysis because they employed different classification methods from the other literature. ${ }^{35,52}$ The results showed that there was no statistically significant difference in the risk of melanoma associated with an elapsed time of $<5$ years (pooled RR: 1.00; 95\% CI: 0.81-1.23; $\mathrm{I}^{2}=0 \%$ ), 5-9 years (pooled RR: 0.99; 95\% CI: $0.81-1.21 ; \mathrm{I}^{2}=0 \%$ ), or $\geq 10$ years (pooled RR: 0.90 ; 95\% CI: $0.52-1.58 ; \mathrm{I}^{2}=86 \%$ ) since the last use (Supplementary figure 3). Seven articles evaluated the relationship between age at first use and incidence of melanoma in women. ${ }^{13,24,25,27,29,35,38}$ Three studies were not included in the analysis because they employed different classification methods from the other literatures, ${ }^{27,29,38}$ and one article was not included in the analysis because the study population was repeated (Table 1). ${ }^{35}$ Compared to non-users, we found that there was no statistically significant risk associated with first use at age 20-24 year (pooled RR: 1.26; 95\% CI: 0.95$1.65 ; \mathrm{I}^{2}=0 \%$ ) or $\geq 25$ years (pooled RR: $1.00 ; 95 \% \mathrm{CI}$ : $0.79-1.27 ; \mathrm{I}^{2}=0 \%$ ) (Supplementary figure 4).

These results suggest that women who first used OC 15-19 years ago were more likely to develop melanoma, 
Table 2 Summary Relative Risks Estimates for Melanoma in Women

\begin{tabular}{|c|c|c|c|c|}
\hline Risk Factors & Total & Pooled RR & P-value & $I^{2}(\%)$ \\
\hline \multicolumn{5}{|l|}{ Oral contraceptive (OC) } \\
\hline Ever versus never & 27 & $0.99(0.90-1.10)$ & 0.87 & 54 \\
\hline Ever versus never in SSM & 6 & $\mathrm{I} .0 \mathrm{I}(0.87-\mathrm{I} .18)$ & 0.87 & 27 \\
\hline Ever versus never in NM & 4 & $0.84(0.55-1.30)$ & 0.44 & 23 \\
\hline Duration: $<2$ years versus never & 5 & $0.87(0.66-1.14)$ & 0.31 & 0 \\
\hline Duration: $<5$ years versus never & 12 & $0.96(0.82-1.13)$ & 0.64 & 59 \\
\hline Duration: $\geq 5$ years versus never & 8 & $1.18(1.07-1.31)$ & 0.001 & 0 \\
\hline Duration: $\geq 10$ years versus never & 10 & $1.25(1.06-1.48)$ & 0.008 & 0 \\
\hline Time since first use: $<15$ years versus never & 2 & $0.96(0.73-1.26)$ & 0.75 & 0 \\
\hline Time since first use: $15-19$ years versus never & 2 & $1.52(1.03-2.24)$ & 0.03 & 0 \\
\hline Time since first use: $\geq 20$ years versus never & 2 & $1.14(0.8 I-1.6 I)$ & 0.44 & 0 \\
\hline Years since last use: $<5$ years versus never & 5 & $1.00(0.81-1.23)$ & 0.99 & 0 \\
\hline Years since last use: $5-9$ years versus never & 5 & $0.99(0.81-1.21)$ & 0.91 & 0 \\
\hline Years since last use: $\geq 10$ years versus never & 3 & $0.90(0.52-1.58)$ & 0.72 & 86 \\
\hline Age at first use: $20-24$ years old versus never & 2 & $1.26(0.95-1.65)$ & 0.11 & 0 \\
\hline Age at first use: $\geq 25$ years old versus never & 3 & $1.00(0.79-1.27)$ & 0.99 & 0 \\
\hline \multicolumn{5}{|l|}{ Hormonal replacement therapy (HRT) } \\
\hline Ever versus never & 16 & $1.12(1.02-1.24)$ & 0.02 & 50 \\
\hline Ever versus never in SSM & 4 & $1.26(1.17-1.37)$ & $<0.00001$ & 0 \\
\hline Ever versus never in NM & 3 & $1.08(0.87-1.33)$ & 0.49 & 0 \\
\hline Estrogen user versus never & 3 & $1.44(1.22-1.69)$ & $<0.0001$ & 0 \\
\hline Estradiol user versus never & 2 & $1.49(1.26-1.77)$ & $<0.00001$ & 0 \\
\hline Estrogen and progestogen user versus never & 3 & $1.08(0.91-1.27)$ & 0.37 & 21 \\
\hline Progestogen user versus never & 2 & $1.58(0.89-2.79)$ & 0.12 & 0 \\
\hline \multicolumn{5}{|l|}{ Reproductive factors } \\
\hline Parity: I birth versus no birth & 7 & $0.94(0.77-1.15)$ & 0.54 & 53 \\
\hline Parity: 2 births versus no birth & 7 & $1.05(0.85-1.30)$ & 0.64 & 69 \\
\hline Parity: 3 births versus no birth & 5 & $0.94(0.74-I .18)$ & 0.58 & 64 \\
\hline Parity: I-2 births versus no birth & 13 & $1.02(0.84-1.25)$ & 0.82 & 77 \\
\hline Parity: 3-4 births versus no birth & 4 & $0.8 I(0.74-0.88)$ & $<0.00001$ & 35 \\
\hline Parity: $\geq 3$ births versus no birth & 11 & $0.87(0.68-1.12)$ & 0.29 & 57 \\
\hline Parity: $\geq 4$ births versus no birth & 5 & $0.90(0.64-1.26)$ & 0.53 & 75 \\
\hline Parity: $\geq 5$ births versus no birth & 4 & $0.66(0.53-0.83)$ & 0.0002 & 27 \\
\hline Age at first birth: $25-29$ versus $<25$ & 5 & $1.14(0.85-1.53)$ & 0.37 & 63 \\
\hline Age at first birth: $\geq 30$ versus $<25$ & 4 & $1.04(0.65-1.65)$ & 0.88 & 72 \\
\hline Age at first birth: $20-24$ versus $<20$ & 2 & $1.16(1.06-1.26)$ & 0.001 & 15 \\
\hline Age at first birth: $25-29$ versus $<20$ & 2 & $1.42(1.30-1.55)$ & $<0.00001$ & 0 \\
\hline Age at first birth: $\geq 30$ versus $<20$ & 3 & $1.34(1.21-1.48)$ & $<0.00001$ & 1 \\
\hline Menopausal status: post versus pre & 7 & $0.96(0.82-I .12)$ & 0.57 & 0 \\
\hline Age at menarche: $13-14$ versus $<13$ & 4 & $1.05(0.90-1.23)$ & 0.51 & 0 \\
\hline Age at menarche: $\geq 15$ versus $<13$ & 4 & $0.85(0.67-1.07)$ & 0.17 & 0 \\
\hline
\end{tabular}

Abbreviations: SSM, superficial spreading melanoma; NM, nodular melanoma; RR, relative risk.

and no association was found between either the elapsed time since last use or the age of first use and the development of melanoma in women. However, due to the small number of studies included in these analyses, these results may be random.

\section{The Use of HRT}

Seventeen articles assessed the risk of melanoma in women who used HRT, ${ }^{14-16,20,27-34,36,38,40,56}$ including one that was not included in the analysis because the study population was repeated. ${ }^{37}$ Of a total of 11 case- 


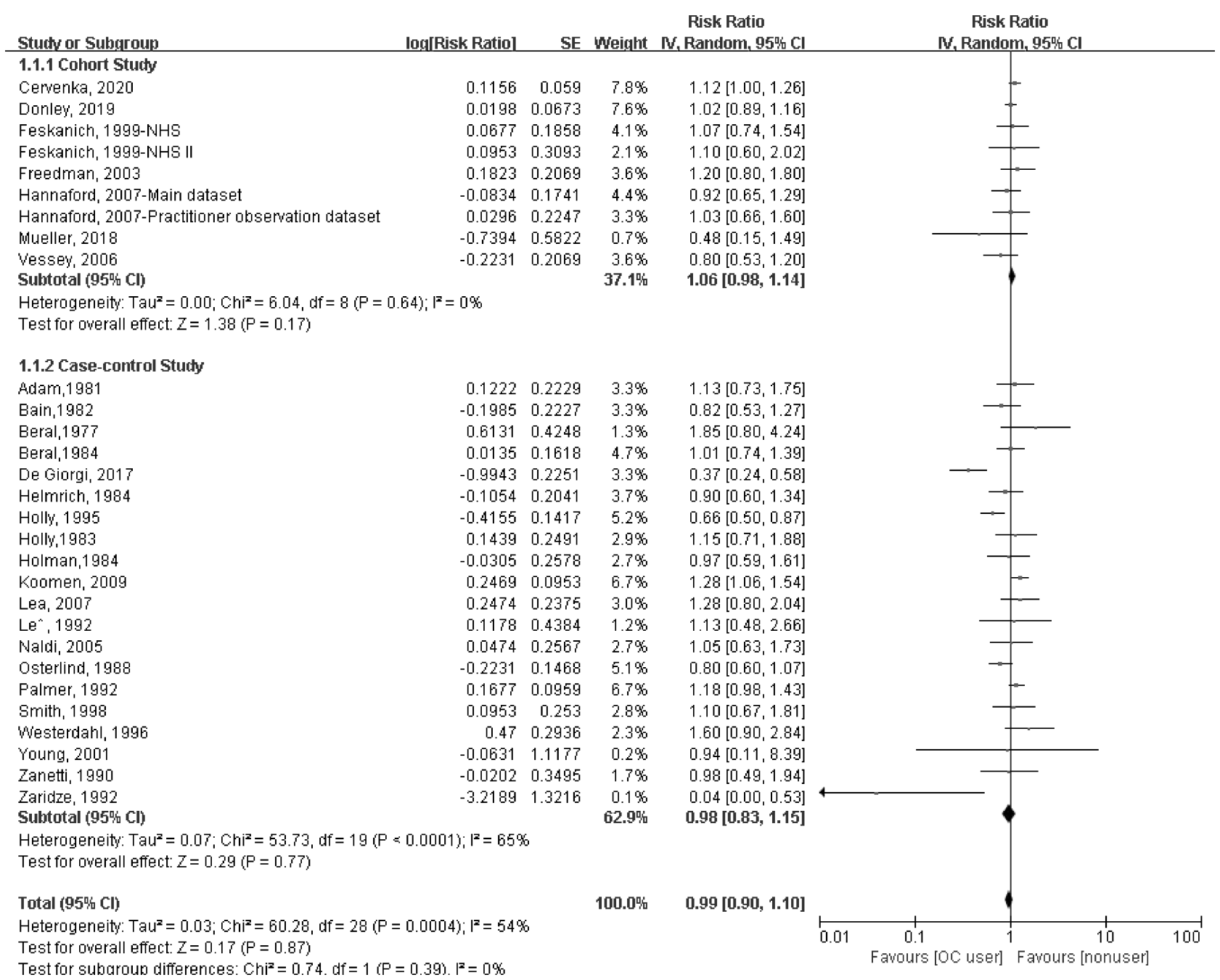

Figure 2 Forest plot of $O C$ use and female melanoma risk.

control studies, ${ }^{14-16,20,27,28,30,32,34,40} 2$ showed a significant risk of melanoma, ${ }^{14,15}$ and 1 showed a reduced risk. ${ }^{40}$ In 5 cohort studies, ${ }^{29,33,36,38,56}$ no significant risk was identified. The combined risk of HRT use for all casecontrol studies was 1.07 (95\% CI: 0.86-1.32; $P=0.56$ ). The combined risk based on all cohort studies was 1.10 (95\% CI: $1.02-1.19 ; P=0.01$ ). The combined pooled risk was 1.12 (95\% CI: $1.02-1.24 ; P=0.02)$, with minimal heterogeneity $\left(\mathrm{I}^{2}=50 \%\right)$ (Table 1 , Figure 4$)$. This suggests that HRT use may increase the incidence of female melanoma. Four articles evaluated the relationship between HRT use and the incidence of different histologic subtypes of female melanoma. ${ }^{15,32,34,37}$ As shown in Table 2, all four of the articles evaluated the relationship between HRT use and the incidence of SSM in women, ${ }^{15,32,34,37}$ and three articles also assessed the risk of $\mathrm{NM}$ in women using HRT. ${ }^{15,34,37}$ The relative risk of SSM for women using HRT was 1.26 (95\% CI: $1.17-1.37 ; P<0.00001)$
(Figure $5 \mathrm{~A}$ ), while the relative risk of $\mathrm{NM}$ was 1.08 (95\% CI: 0.87-1.33; $P=0.49$ ) (Figure 5B), and there was no heterogeneity $\left(\mathrm{I}^{2}=0 \%\right.$ for both). This suggests that the use of HRT may increase the risk of SSM in women but has no effect on NM.

We also investigated the effect of the active substances contained in the HRT on the incidence of melanoma in women. However, only four articles provided the active substances involved. ${ }^{15,16,38,56}$ As shown in Table 2, the relative risk of melanoma for women using estrogen was 1.44 (95\% CI: $1.22-1.69 ; P<0.0001 ; \mathrm{I}^{2}=0 \%$ ), the relative risk of melanoma for women using estradiol was 1.49 (95\% CI: $1.26-1.77 ; \quad P<0.00001 ; I^{2}=0 \%$ ), while there was no statistically significant risk of melanoma in women with estrogen and progestogen (pooled RR: 1.08; 95\% CI: $0.91-1.27 ; P=0.37 ; \mathrm{I}^{2}=21 \%$ ) and progestogen (pooled RR: $1.58 ; 95 \%$ CI: $0.89-2.79 ; P=0.12 ; I^{2}=0 \%$ ) (Supplementary figure 5). This suggests that the use of 


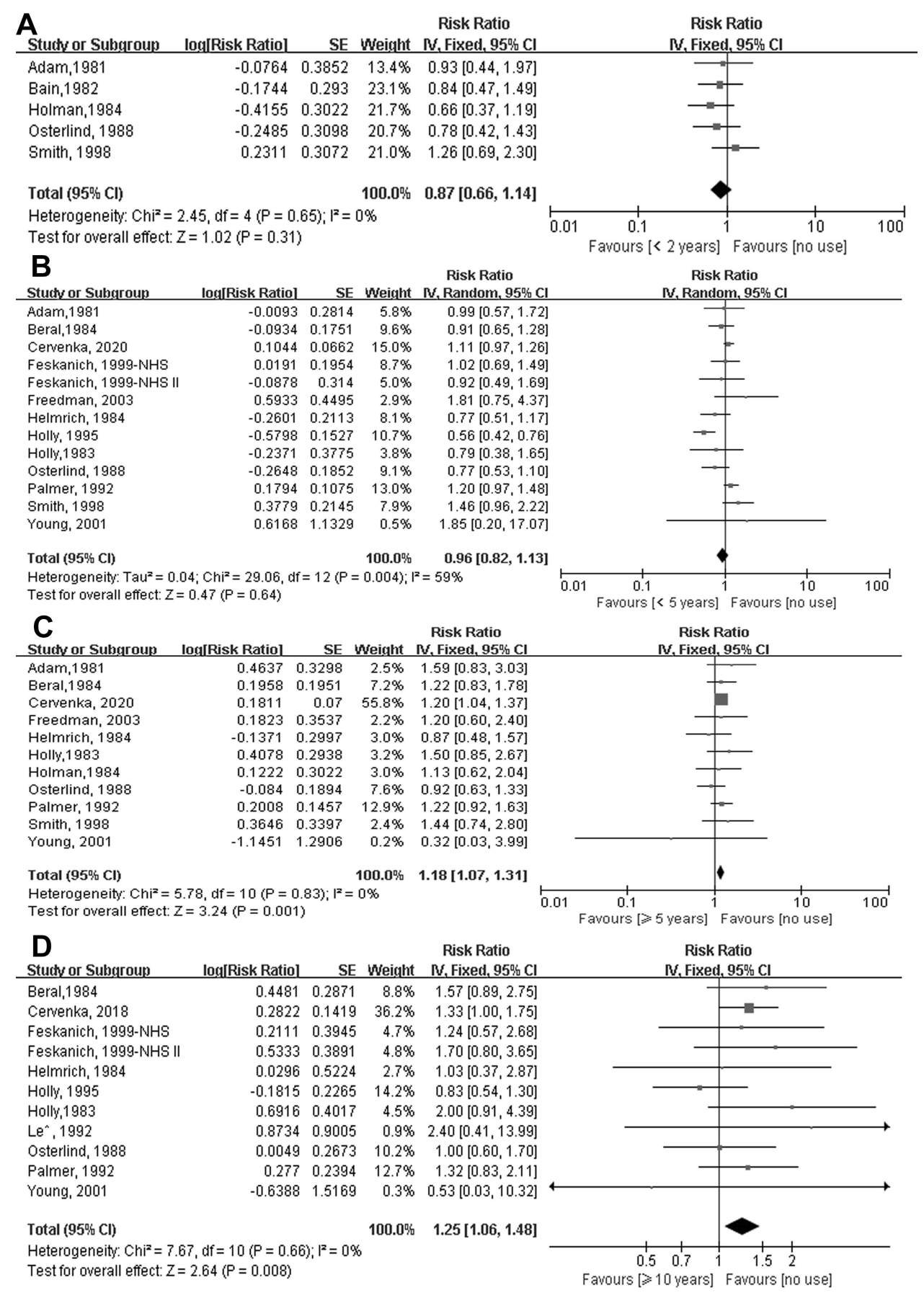

Figure 3 Forest plot of duration of $\mathrm{OC}$ use and female melanoma risk. (A) $<2$ years, (B) $<5$ years, (C) $\geq 5$ years, (D) $\geq 10$ years.

estrogen and estradiol may increase the risk of SSM in women, while progestogen does not.

\section{Reproductive Factors}

A total of 19 studies assessed the relationship between reproductive factors and female melanoma. ${ }^{16,21,23,24,26-}$ $32,34,36,41-43,51,54,55$
Eighteen articles assessed the effect of parity on female melanoma, ${ }^{16,21,23,24,26-28,30,32,36,41-43,51,54,55} \quad$ including three that were not included in the analysis because their classifications were different from the other studies or no data was available. ${ }^{36,42,55}$ As shown in Table 2, compared with a parity of 0 , the relative risk of melanoma in women with 1 (pooled RR: $0.94 ; 95 \%$ CI: $0.77-1.15 ; \mathrm{I}^{2}=53 \%$ ), 


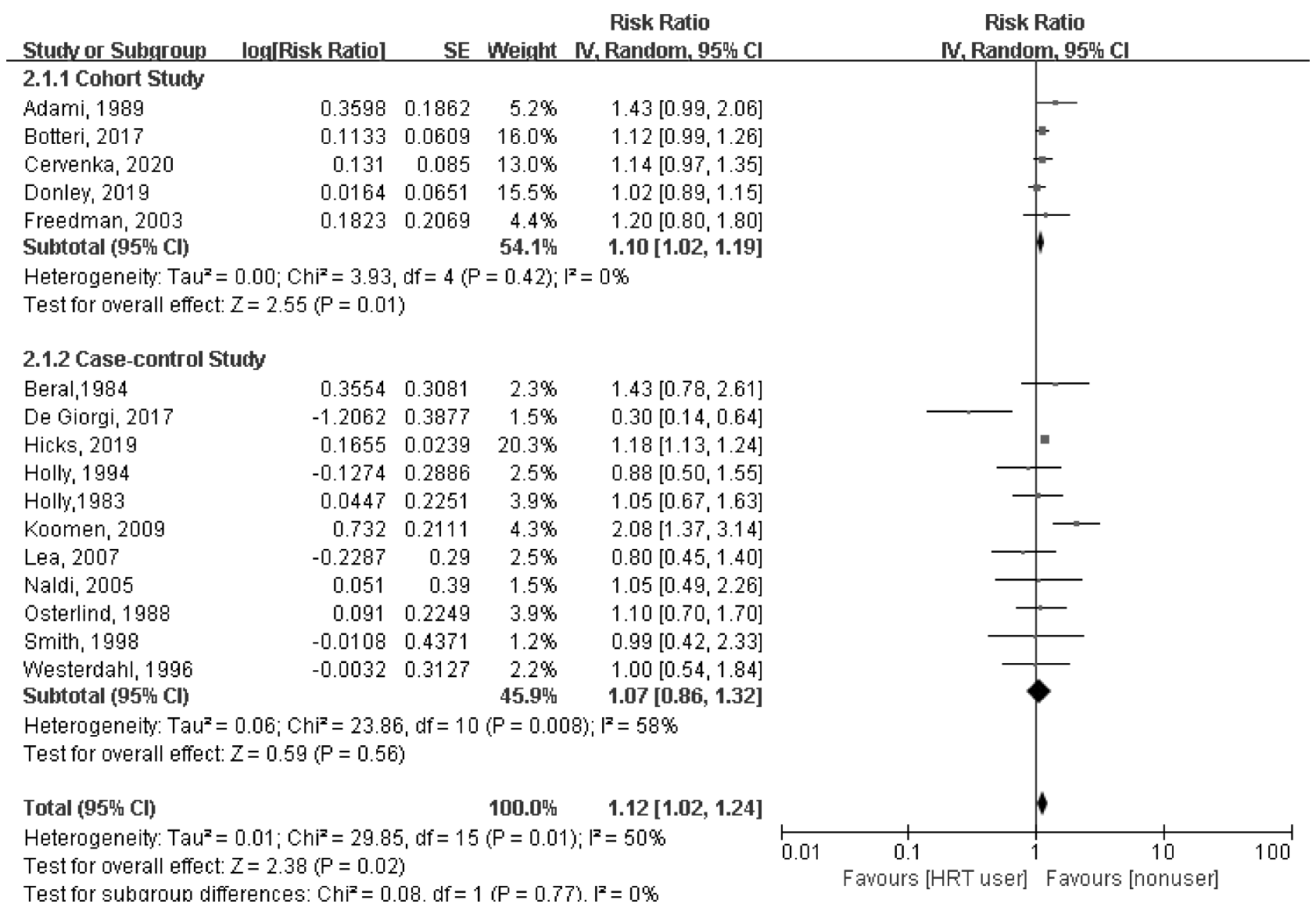

Figure 4 Forest plot of HRT use and female melanoma risk.

\begin{tabular}{|c|c|c|c|c|c|c|c|c|}
\hline $\begin{array}{l}\text { A } \\
\text { Stucty or Subqroup }\end{array}$ & log[Risk Ratio] & $\mathrm{SE}$ & Weight & $\begin{array}{c}\text { Risk Ratio } \\
\text { N, Fixed, 95\% } \mathrm{Cl}\end{array}$ & & $\begin{array}{r}\text { Risk } \\
\text { W, Fixed }\end{array}$ & $\begin{array}{l}\text { Ratio } \\
\text { d, } 95 \% \mathrm{Cl}\end{array}$ & \\
\hline Cervenka, 2019 & 0.3506 & 0.1574 & $6.5 \%$ & $1.42[1.04,1.93]$ & & & & \\
\hline Hicks, 2019 & 0.2316 & 0.0424 & $89.8 \%$ & $1.26[1.16,1.37]$ & & & & \\
\hline Holly, 1994 & 0.0327 & 0.3357 & $1.4 \%$ & $1.03[0.54,2.00]$ & & & & \\
\hline Holly,1983 & 0.1465 & 0.265 & $2.3 \%$ & $1.16[0.69,1.95]$ & & & & \\
\hline Total $(95 \%$ Cl) & & & $100.0 \%$ & $1.26[1.17,1.37]$ & & & 1 & \\
\hline $\begin{array}{l}\text { Heterogeneity: } \mathrm{Chi}^{2}= \\
\text { Test for overall effect }\end{array}$ & $\begin{array}{l}1.02, d f=3(P=0 \\
Z=5.84(P<0.00\end{array}$ & $.80) ; 1^{2}=$ & & & 0.01 & $\begin{array}{c}0.1 \\
\text { Favours [HRT use] }\end{array}$ & Favours [no use] & 100 \\
\hline $\begin{array}{l}\text { B } \\
\text { Stucty or Subqroup }\end{array}$ & loglRisk Ratio] & SE & Weight & $\begin{array}{c}\text { Risk Ratio } \\
\text { N, Fixed, 95\% } \mathrm{Cl}\end{array}$ & & $\begin{array}{r}\text { Risk } \\
\text { P, Fixed }\end{array}$ & $\begin{array}{l}\text { Ratio } \\
\text { d, } 95 \% \text { Cl }\end{array}$ & \\
\hline Cervenka, 2019 & -0.5752 & 0.6745 & $2.6 \%$ & $0.56[0.15,2.11]$ & & & & \\
\hline Hicks, 2019 & -0.1958 & 0.5874 & $3.4 \%$ & $0.82[0.26,2.60]$ & & & & \\
\hline Holly, 1994 & 0.1028 & 0.1119 & $94.0 \%$ & $1.11[0.89,1.38]$ & & & & \\
\hline Total $(95 \%$ Cl) & & & $100.0 \%$ & $1.08[0.87,1.33]$ & & & & \\
\hline \multicolumn{5}{|c|}{$\begin{array}{l}\text { Heterogeneity: } \mathrm{Chi}^{2}=1.20, \mathrm{df}=2(P=0.55) ; I^{2}=0 \% \\
\text { Test for overall effect: } Z=0.69(P=0.49)\end{array}$} & 0.01 & $\begin{array}{c}0.1 \\
\text { Favours [HRT use] }\end{array}$ & $\begin{array}{l}10 \\
\text { Favours [no use] }\end{array}$ & $100^{\circ}$ \\
\hline
\end{tabular}

Figure 5 Forest plot of HRT use and different histological subtypes of female melanoma risk. (A) SSM, (B) NM.

two (pooled RR: $1.05 ; 95 \%$ CI: $0.85-1.30 ; \mathrm{I}^{2}=69 \%$ ), three (pooled RR: 0.94; 95\% CI: $0.74-1.18 ; \mathrm{I}^{2}=64 \%$ ), $1-2$ (pooled RR: 1.02; 95\% CI: $0.84-1.25 ; \mathrm{I}^{2}=77 \%$ ), $\geq 3$ (pooled RR: 0.87; 95\% CI: $0.68-1.12 ; \mathrm{I}^{2}=57 \%$ ), or $\geq 4$ (pooled $\mathrm{RR}=0.90 ; 95 \% \mathrm{CI}: 0.64-1.26 ; \mathrm{I}^{2}=75 \%$ ) births had no statistical significance (Figure 6A-D, F and G). 


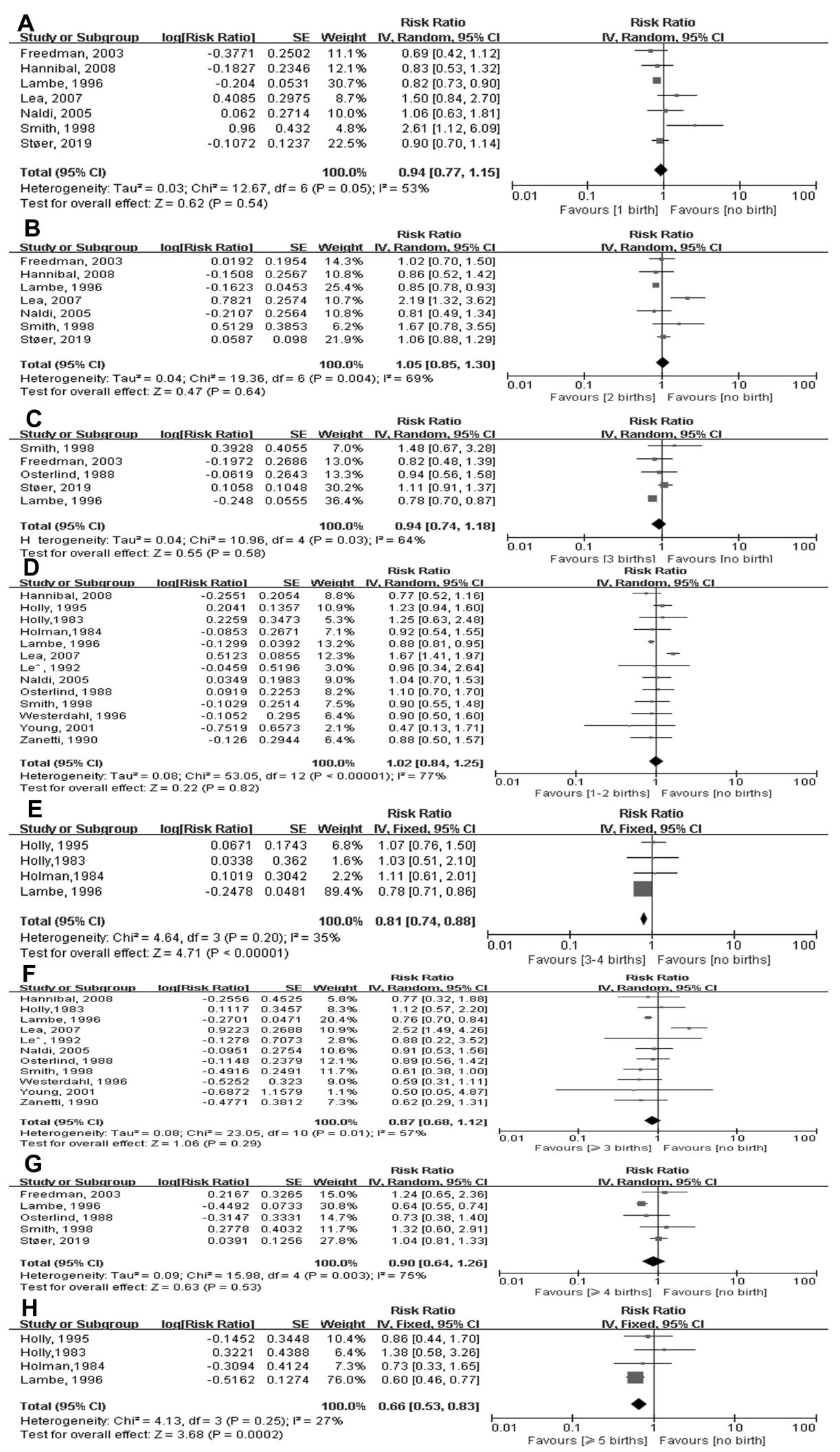

Figure 6 Forest plot of parity and female melanoma risk. (A) I birth, (B) 2 births, (C) 3 births, (D) I-2 births, (E) $3-4$ births, (F) $\geq 3$ births, (G) $\geq 4$ births, (H) $\geq 5$ births. 
However, women with 3-4 births (pooled RR: 0.81; 95\% CI: $0.74-0.88 ; \quad I^{2}=35 \%$ ) (Figure $6 \mathrm{E}$ ) and $\geq 5$ births (pooled RR: $0.66 ; \quad 95 \%$ CI: $0.53-0.83 ; \quad I^{2}=27 \%$ ) (Figure $6 \mathrm{H}$ ) had a reduced risk of melanoma. This suggests that women with more births may be less likely to develop melanoma.

Thirteen articles assessed the effect of age at first birth on female melanoma, ${ }^{23,26,28-30,32,36,41-43,51,54,55}$ including six that were not included in the analysis because their classification was different from the other studies or no data were available. ${ }^{26,30,32,43,51,54}$ As shown in Table 2, there was no statistically significant risk of melanoma in women aged 25-29 years (pooled RR: 1.14; 95\% CI: $0.85-1.53 ; \mathrm{I}^{2}=63 \%$ ) and $\geq 30$ years (pooled RR: 1.04; 95\% CI: $0.65-1.65 ; \mathrm{I}^{2}=72 \%$ ) compared with those aged $<25$ at their first birth (Figure $7 \mathrm{~A}$ and B). However,

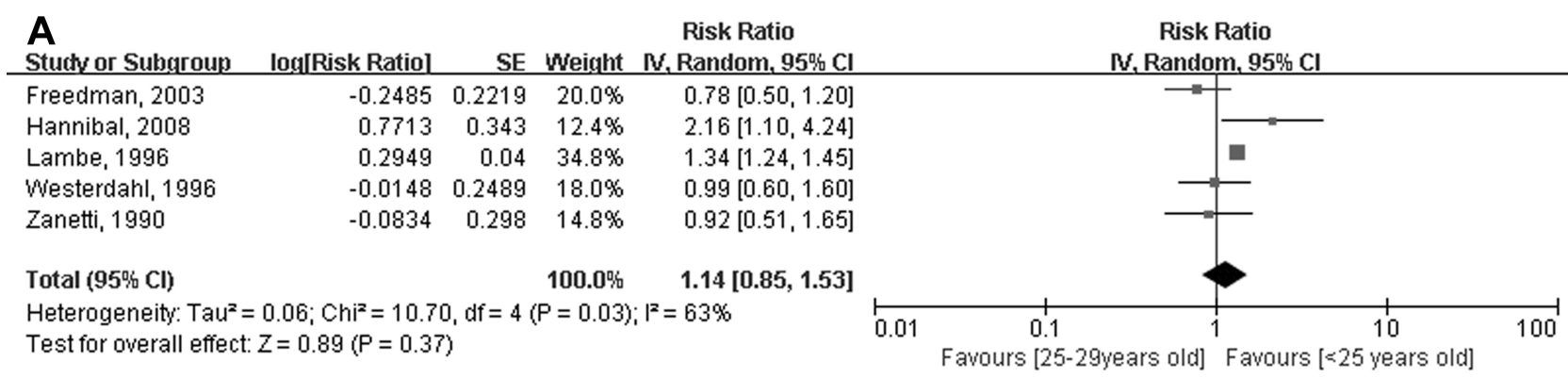

B

Risk Ratio

\begin{tabular}{|c|c|c|c|c|c|c|c|c|}
\hline Stucty or Subgroup & log[Risk Ratio] & SE & Weight & W, Random, 95\% Cl & & N, Rando & $\mathrm{om}, 95 \% \mathrm{Cl}$ & \\
\hline Freedman, 2003 & -0.5447 & 0.2971 & $23.3 \%$ & $0.58[0.32,1.04]$ & & $\longrightarrow$ & & \\
\hline Hannibal, 2008 & 0.709 & 0.3474 & $20.5 \%$ & $2.03[1.03,4.01]$ & & & & \\
\hline Lambe, 1996 & 0.2285 & 0.0603 & $36.0 \%$ & $1.26[1.12,1.41]$ & & & E & \\
\hline Zanetti, 1990 & -0.3285 & 0.3537 & $20.2 \%$ & $0.72[0.36,1.44]$ & & & . & \\
\hline Total (95\% & & & $100.0 \%$ & $1.04[0.65,1.65]$ & & & & \\
\hline $\begin{array}{l}\text { Heterogeneity: Tau }{ }^{2} \\
\text { Test for overall effec }\end{array}$ & $\begin{array}{l}0.15 ; \mathrm{Chi}^{2}=10.88 \\
Z=0.15(P=0.88\end{array}$ & $d f=3$ & $P=0.01)$ & $F^{2}=72 \%$ & 0.01 & $\begin{array}{l}0.1 \\
\text { Favours }[\geqslant 30]\end{array}$ & $\begin{array}{lc}1 & 10 \\
\text { Favours }[<25]\end{array}$ & 100 \\
\hline
\end{tabular}

C

Risk Ratio

Studv or Subqroup log[Risk Ratiol SE Weight N, Fixed, 95\% Cl

$\begin{array}{lllll}\text { Lambe, } 1996 & 0.1044 & 0.0584 & 58.0 \% & 1.11\end{array}[0.99,1.24]$

$\begin{array}{lllll}\text { Neale, } 2005 & 0.2021 & 0.0686 & 42.0 \% & 1.22[1.07,1.40]\end{array}$

Total (95\% Cl)

$100.0 \% 1.16[1.06,1.26]$

Heterogeneity: $\mathrm{Chi}^{2}=1.18, \mathrm{df}=1(\mathrm{P}=0.28) ; \mathrm{I}^{2}=15 \%$

Test for overall effect: $Z=3.27(P=0.001)$

D

\begin{tabular}{|c|c|c|c|c|}
\hline Stucty or Subqroup & log[Risk Ratio] & SE & Weight & N, Fixed, 95\% Cl \\
\hline Lambe, 1996 & 0.3293 & 0.0583 & $59.3 \%$ & $1.39[1.24,1.56]$ \\
\hline Neale, 2005 & 0.3771 & 0.0704 & $40.7 \%$ & $1.46[1.27,1.67]$ \\
\hline Total $(95 \%$ Cl) & & & $100.0 \%$ & $1.42[1.30,1.5$ \\
\hline
\end{tabular}

Test for overall effect: $Z=7.77(P=0.00001)$

\section{Risk Ratio}

Study or Subgroup log[Risk Ratiol SE Weight N, Fixed, 95\% Cl

$\begin{array}{lllll}\text { Donley, } 2019 & 0.0742 & 0.1627 & 9.9 \% & 1.08[0.78,1.48]\end{array}$

$\left.\begin{array}{lllll}\text { Lambe, } 1996 & 0.3197 & 0.0721 & 50.4 \% & 1.38\end{array}\right]$

Neale, 2005

Total (95\% Cl)

Heterogeneity: $\mathrm{Chi}^{2}=2.03, \mathrm{df}=2(\mathrm{P}=0.36) ; \mathrm{I}^{2}=1 \%$

Test for overall effect: $Z=5.74$ ( $P<0.00001$ )
Risk Ratio

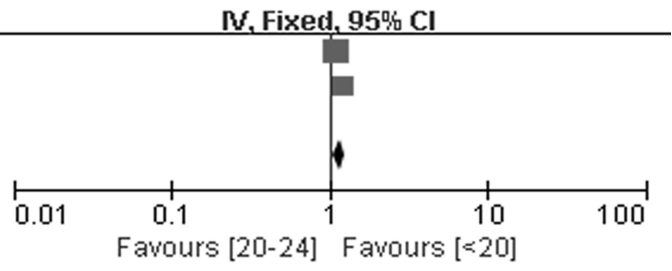

Risk Ratio N, Fixed, 95\% Cl

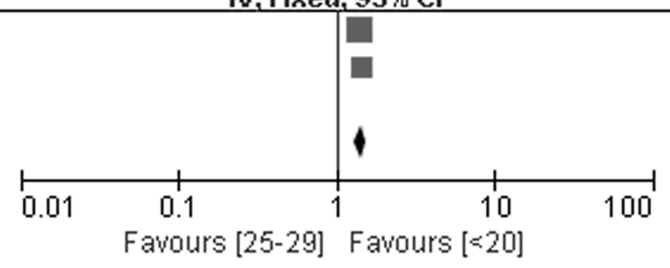

Risk Ratio

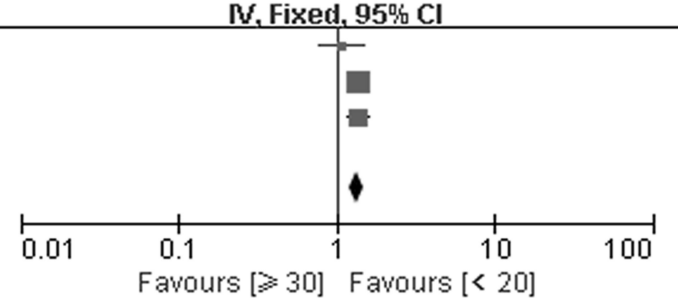

Figure 7 Forest plot of age at first birth and femalemelanoma risk. (A) $25-29$ vs. $<25$, (B) $\geq 30$ vs. $<25$, (C) $20-24$ vs. $<20$, (D) $25-29$ vs. $<20$, (E) $\geq 30$ vs. $<20$. 
Table 3 Heterogeneity Analyses: Relative Risks (RR) Estimates by Subgroup Analyses

\begin{tabular}{|c|c|c|c|c|c|c|c|c|}
\hline \multirow[t]{2}{*}{ Groups } & \multicolumn{4}{|c|}{ OC User (“Ever Use”) } & \multicolumn{4}{|c|}{ HRT User (“Ever Use”) } \\
\hline & $\begin{array}{l}\text { No. of } \\
\text { Studies }\end{array}$ & $\mathbf{R R}(95 \% \mathrm{CI})$ & P-value & $I^{2}(\%)$ & $\begin{array}{l}\text { No. of } \\
\text { Studies }\end{array}$ & $\mathbf{R R}(95 \% \mathrm{Cl})$ & P-value & $I^{2}(\%)$ \\
\hline Total & 27 & $0.99(0.90-I .10)$ & 0.87 & 54 & 16 & $1.12(1.02-1.24)$ & 0.02 & 50 \\
\hline Study design & & & & & & & & \\
\hline Cohort & 7 & $1.06(0.98-1.14)$ & 0.17 & 0 & 5 & $1.10(1.02-1.19)$ & 0.01 & 0 \\
\hline Case-control & 20 & $0.98(0.83-1.15)$ & 0.77 & 65 & 11 & $1.07(0.86-1.32)$ & 0.56 & 58 \\
\hline \multicolumn{9}{|l|}{ Geographic area } \\
\hline US & 11 & $1.03(0.90-1.18)$ & 0.7 & 40 & 6 & $1.02(0.91-1.14)$ & 0.79 & 0 \\
\hline UK(OC user) or Sweden(HRT user) & 3 & $0.95(0.78-1.16)$ & 0.62 & 0 & 2 & $1.30(0.95-1.78)$ & 0.1 & 0 \\
\hline $\begin{array}{l}\text { Australia(OC user) or Denmark(HRT } \\
\text { user) }\end{array}$ & 3 & $1.00(0.77-1.31)$ & 1 & 0 & 2 & $1.18(1.13-1.24)$ & $<0.00001$ & 0 \\
\hline Italy & 3 & $0.7 I(0.34-I .47)$ & 0.35 & 82 & 2 & $0.56(0.33-0.96)$ & 0.03 & 81 \\
\hline Other countries & 7 & $\mathrm{I} .07(0.87-\mid .3 \mathrm{I})$ & 0.55 & 63 & 4 & $1.17(1.06-1.28)$ & 0.001 & 64 \\
\hline \multicolumn{9}{|l|}{$\begin{array}{l}\text { Whether the confounders were } \\
\text { corrected for }\end{array}$} \\
\hline Adjusted RRs & 18 & $1.05(0.97-1.14)$ & 0.2 & 16 & 13 & $1.13(1.05-1.21)$ & 0.0007 & 25 \\
\hline Crude RRs & 9 & $0.88(0.66-I .18)$ & 0.39 & 77 & 3 & $0.89(0.38-2.11)$ & 0.8 & 86 \\
\hline \multicolumn{9}{|l|}{$\begin{array}{l}\text { Whether education was corrected } \\
\text { for }\end{array}$} \\
\hline Yes & 6 & $1.09(1.00-1.18)$ & 0.05 & 0 & 6 & $1.07(1.00-1.16)$ & 0.06 & 0 \\
\hline No & 21 & $0.95(0.83-1.09)$ & 0.48 & 61 & 10 & $1.17(0.96-1.43)$ & 0.11 & 59 \\
\hline \multicolumn{9}{|l|}{$\begin{array}{l}\text { Whether the daylight exposure } \\
\text { factor was corrected for }\end{array}$} \\
\hline Yes & 9 & $1.03(0.89-1.19)$ & 0.72 & 25 & 7 & $1.07(0.99-1.17)$ & 0.09 & 0 \\
\hline No & 18 & $0.97(0.84-I . I I)$ & 0.62 & 63 & 9 & I.I4(0.95-I.37) & 0.15 & 67 \\
\hline
\end{tabular}

Abbreviations: OC, oral contraceptives; HRT, hormone replacement therapy.

women aged 20-24 years (pooled RR: 1.16; 95\% CI: 1.06-1.26; $\mathrm{I}^{2}=15 \%$ ), 25-29 years (pooled RR: 1.42; 95\% CI: $1.30-1.55 ; \mathrm{I}^{2}=0 \%$ ), and $\geq 30$ years (pooled RR: 1.34 ; 95\% CI: $1.21-1.48 ; \mathrm{I}^{2}=1 \%$ ) showed significant risk compared to those aged less than 20 years at first birth (Figure 7C-E). This suggests that women who give birth for the first time at $\geq 20$ years of age have an increased risk of melanoma compared to those who first give birth aged $<20$ years.

Seven articles evaluated the effects of menopausal status. ${ }^{16,27,29,30,34,43,55}$ As shown in Table 2, the combined risk of melanoma in postmenopausal women was 0.96 (95\% CI: $0.82-1.12 ; \mathrm{I}^{2}=0$ ) compared with premenopausal women (Supplementary figure 6). This suggests that menopause has no effect on the incidence of melanoma in women.

Nine articles evaluated the effect of age at menarche, ${ }^{16,21,24,29,34,36,43,51,55}$ five of which were not included in this analysis due to stratification. ${ }^{24,29,34,36,43}$ As shown in Table 2, the risk of melanoma in women with menarche aged 13-14 years (pooled RR: 1.05; 95\% CI: $0.90-1.23 ; \mathrm{I}^{2}=0 \%$ ) and $\geq 15$ years (pooled RR: 0.85 ; 95\% CI: $0.67-1.07 ; \mathrm{I}^{2}=0 \%$ ) was not statistically significant compared with menarche age $<13$ (Supplementary figure 7). This suggests that age at menarche has no effect on melanoma in women.

\section{Heterogeneity Analysis}

Investigation of heterogeneity was carried out for factors with the highest number of studies that allowed subgroup analyses, including the use of OC and HRT, to identify factors that could have an impact on the inter-study heterogeneity.

Table 3 shows the results of subgroup analyses, stratified by study design type, geographic area, whether confounders were corrected for, whether education was corrected for, and 
whether daylight exposure was corrected for. The use of OC was not associated with a risk of melanoma in women in most subgroups, and only after adjustment for education was there a critical statistical significance for an increased risk of melanoma (RR: $1.09 ; 95 \%$ CI: $1.00-1.18 ; \mathrm{I}^{2}=0 \%$ ). The use of HRT showed an increased risk of melanoma in cohort studies (RR: $1.10 ; 95 \%$ CI: $1.02-1.19 ; P=0.01$ ), and heterogeneity between cohort studies $\left(\mathrm{I}^{2}=0 \%\right)$ was significantly lower than case-control studies $\left(\mathrm{I}^{2}=58 \%\right)$. There was no statistically significant difference in the US (RR: $1.02 ; 95 \%$ CI: $0.91-$ $1.14 ; \mathrm{I}^{2}=0 \%$ ) and Sweden (RR: 1.30 ; 95\% CI: 0.95-1.78; $\mathrm{I}^{2}=0 \%$ ), but we have observed a protective effect of HRT on melanoma in women in Italy (RR: 0.56 ; $95 \%$ CI: 0.33-0.96; $\mathrm{I}^{2}=81 \%$ ), the risk of melanoma increased significantly after HRT use in Denmark (RR: 1.18; 95\% CI: 1.13-1.24; $\mathrm{I}^{2}<0.00001$ ) and other countries (RR: 1.17 ; 95\% CI: 1.06 $1.28 ; \mathrm{I}^{2}=64 \%$ ). In addition, by looking at the types of adjustments, we found that after adjusting for confounders, HRT users had a higher risk of melanoma (RR: 1.13 ; 95\% CI: $\left.1.05-1.21 ; \mathrm{I}^{2}=25 \%\right)$. However, we observed that there was no increased risk of melanoma in women who used HRT after adjusting for educational (RR: $1.07 ; 95 \%$ CI: $1.00-1.16$; $\mathrm{I}^{2}=0 \%$ ) or daylight factors (RR: $1.07 ; 95 \%$ CI: $0.99-1.17$; $\mathrm{I}^{2}=0 \%$ ). These results suggest that educational factors and daylight factors may be the sources of heterogeneity.

\section{Sensitivity Analysis}

Sensitivity analyses investigated the impact of a single study on overall risk estimates by omitting one study in each turn, to demonstrate that most overall risk estimates were not significantly modified by any single study. However, we identified one study ${ }^{35}$ that had a significant impact on the overall relative risk associated with using OC for a duration $\geq 10$ years. After excluding this study, the overall relative risk changed from 1.25 (95\% CI: 1.06-1.48; $\left.\mathrm{I}^{2}=0 \%\right)$ to 1.21 ( $95 \% \mathrm{CI}: 0.98-1.49 ; \mathrm{I}^{2}=0 \%$ ), and the heterogeneity remained at 0 . We also identified one study that had a significant impact on the overall relative risk of HRT use. ${ }^{15}$ When this study was excluded, the overall relative risk changed from 1.12 (95\% CI: $\left.1.02-1.24 ; \mathrm{I}^{2}=50 \%\right)$ to 1.11 (95\% CI: $0.98-1.26 ; \mathrm{I}^{2}=48 \%$ ). One study had a significant impact on the overall relative risk associated with 1 birth. ${ }^{27}$ After excluding this study, the overall relative risk changed from $0.94\left(95 \% \mathrm{CI}: 0.77-1.15 ; \mathrm{I}^{2}=53 \%\right)$ to 0.84 (95\% CI: $0.77-0.92 ; \mathrm{I}^{2}=15 \%$ ). One study had a significant impact on the overall relative risk of 3 births. ${ }^{43}$ After excluding this study, the overall relative risk changed from 0.94 (95\% CI: $\left.0.74-1.18 ; \mathrm{I}^{2}=64 \%\right)$ to 0.80 (95\% CI: $0.72-$
$0.88 ; \mathrm{I}^{2}=0 \%$ ). One study had a significant impact on the overall relative risk of $1-2$ births and $\geq 3$ births. ${ }^{31}$ After excluding this study, the overall relative risk changed from 1.02 (95\% CI: $\left.0.84-1.25, \mathrm{I}^{2}=77 \%\right)$ to $0.91(95 \%$ CI: $0.85-$ $\left.0.97 ; \mathrm{I}^{2}=0 \%\right)$ and from 0.87 (95\% CI: $0.68-1.12 ; \mathrm{I}^{2}=57 \%$ ) to 0.76 ( $95 \%$ CI: $0.70-0.83 ; \mathrm{I}^{2}=0 \%$ ), respectively. One study had a significant impact on the overall relative risks of 3-4 births and $\geq 5$ births. ${ }^{41}$ After excluding this study, the overall relative risks were changed from $0.81(95 \% \mathrm{CI}$ : $\left.0.74-0.88 ; \mathrm{I}^{2}=35 \%\right)$ to 1.07 (95\% CI: $\left.0.82-1.41 ; \mathrm{I}^{2}=0 \%\right)$ and from 0.66 (95\% CI: $\left.0.53-0.83 ; \mathrm{I}^{2}=27 \%\right)$ to $0.93(95 \%$ CI: $0.60-1.45 ; \mathrm{I}^{2}=0 \%$ ), respectively.

\section{Publication Bias}

There was no evidence of publication bias with regard to OC use, HRT use, or reproductive factors in relation to melanoma risk, as assessed by the Begg rank correlation test and the Egger linear regression test (all $\mathrm{P}>0.05$ ) (Figures 8 and 9).

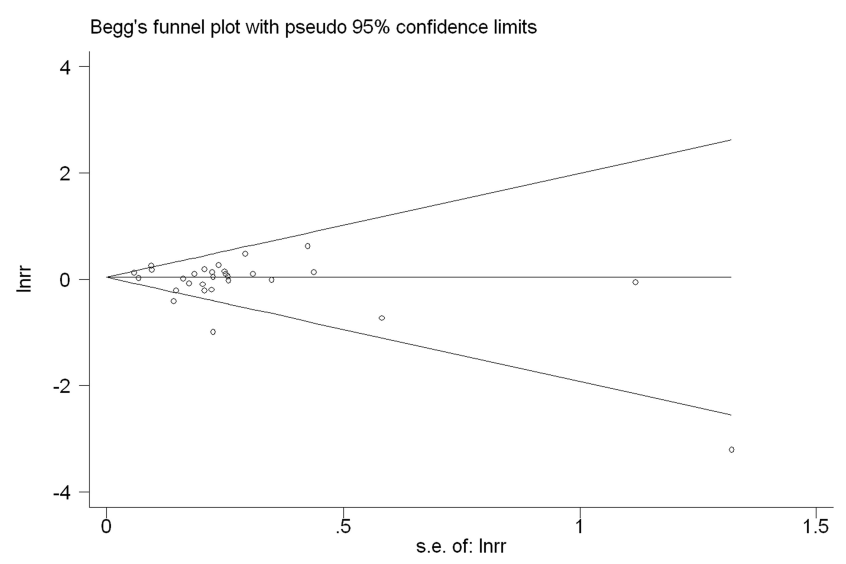

Figure 8 The funnel plot of OC use and female melanoma risk.

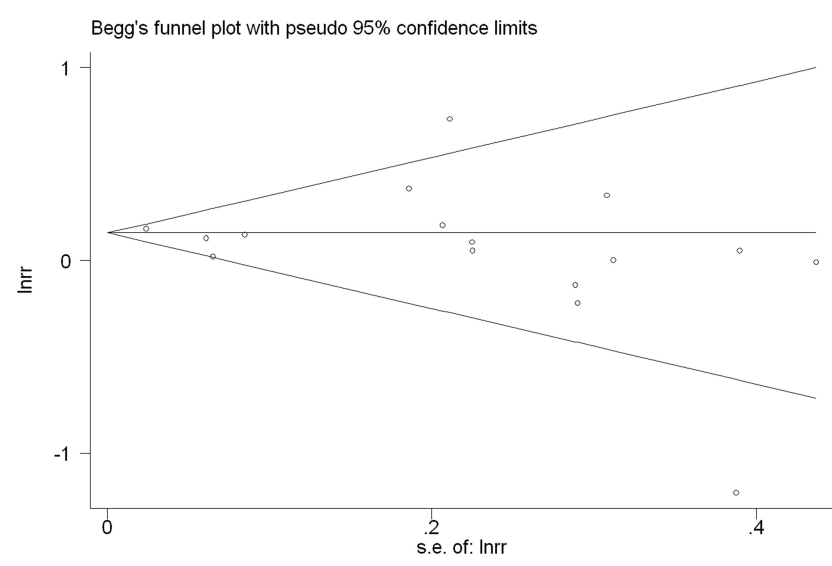

Figure 9 The funnel plot of HRT use and female melanoma risk. 


\section{Discussion}

Estrogen has long been thought to play a role in the development of female melanoma, but the results from observational studies are controversial. These studies included questions about the use of OC and HRT, age at menarche, parity, age at first birth, and menopausal status, all of which reflect the effects of exogenous and endogenous hormones. This meta-analysis of all available prospective cohort and case-control studies suggests that using $\mathrm{OC}$ for more than 5 years, OC use 15-19 years prior to study enrollment, HRT use, and age at first birth of $\geq 20$ years may be associated with an increased risk of melanoma in women. We also observed that HRT use was particularly associated with an increased risk of SSM, but not NM, and estrogen and estradiol may be the main active agents that contribute to the increased risk of melanoma. Our analysis further indicated that an increased number of births may reduce a woman's risk of melanoma. However, any use of $\mathrm{OC}$, years since the last use of OC, age at the first use of OC, menopausal status, and age at menarche were not associated with the incidence of melanoma in women.

Three previous meta-analyses of case-control studies concluded that OC use does not affect a woman's risk of melanoma. ${ }^{58-60}$ A meta-analysis by Gandini et al concluded that OC and HRT do not increase the risk of melanoma, and that exogenous female hormones do not increase the risk of melanoma. In this analysis, there was a significant correlation between melanoma and parity and age at first birth. ${ }^{61}$

Our meta-analysis, updating the previous summary analysis, confirmed that OC use does not increase the risk of melanoma, and that the occurrence of melanoma is associated with parity and age at first birth. In addition, we observed that women who first used OC 15-19 years prior to the study were more likely to develop melanoma, while the age at first OC use, years since the last use, age at menarche, and menopausal status were not associated with an increased risk of melanoma. However, the sensitivity analysis showed that the results of many subgroups related to parity were sensitive, so the reliability of the conclusions from this part of the analysis was insufficient. Further large prospective studies are necessary to clarify the relationship between parity and melanoma. Although our pooled results showed an increased risk of melanoma in women for whom 15-19 years had elapsed since first OC use, the result was only a summary of two studies, so the result was not conclusive. In contrast to the results of the previous meta-analysis, we found that women using OC for $\geq 5$ years and $\geq 10$ years have an increased risk of melanoma; however, sensitivity analysis showed that one study had a strong impact on the results of the summary analysis for duration of OC use $\geq 10$ years. ${ }^{35}$ Therefore, the reliability of these results is questionable and deserves further large-scale prospective studies to verify these findings.

We also found that the use of HRT increased the risk of melanoma in women, especially SSM, and estrogen and estradiol may be the main active agents that contribute to the increased risk of melanoma, but that the effect of HRT was not statistically significant in the subgroup analysis of case-control studies, the US, the UK, and subgroups that adjusted for sun exposure factors. We therefore speculate that the results may be due to a combination of sun exposure factors. Moreover, recent studies have shown that endogenous hormone exposure and its correlation with UV exposure in women from menarche to menopause is an important research area. ${ }^{36}$

Our study pooled all available studies to date, including cohort studies and case-control studies. Risk factors considered in our analysis included the use of OC, duration of use, years since last use, time since the first use, age at first use, the use of HRT, age at menarche, parity, age at first birth, and menopausal status, which makes our meta-analysis more comprehensive, specific, and detailed than previous meta-analyses. Another advantage of this meta-analysis is the inclusion of a multivariate adjusted risk model, and that we performed subgroup analysis for the two groups with the largest number of articles, including study design type, geographic area, whether confounders were corrected for, whether education was corrected for, and whether daylight exposure was corrected for, to minimize the influence of potential confounders on the results so as to more truly reflect the influence of target risk factors on the outcome.

Although the high-quality assessment scores indicate that most of the included studies were of high quality, we acknowledge that this meta-analysis has some limitations and the results should therefore be interpreted with caution. We hypothesize that the potential sources of bias are as follows: (1) the inheritance of unmeasured or uncontrolled confounders from the original study was a concern in this meta-analysis. Although most risk estimates are derived from multivariate models, these studies do not consistently adjust for potential risk factors. Some of the risk estimates are not adjusted for multivariate factors or 
calculated directly from the raw data in the paper. It is therefore uncertain whether the observed increase in risk estimates is merely a response to the target risk factors and not to other confounders; (2) research based on study design also has inherent limitations. The type of study design included both prospective and retrospective studies. There is a possibility of recall bias in retrospective studies because information about the use of OC or HRT was collected after the onset of the disease; (3) another limitation of this meta-analysis was the fact that we combined ORs, RRs and HRs because of the small number of studies included. The presenting risk estimates for ORs and HRs were combined in the meta-analysis on an assumption of constant risk over time and acknowledging that, despite melanoma being a rare disease, the ORs will slightly overestimate the true relative risk; (4) large heterogeneity exists between different studies. Heterogeneity may be due to differences in exposure definitions, exposure ranges, assessment methods, and population characteristics between studies. For example, there are differences in the type, dose, duration, and frequency of use of OC or HRT. The studies included in this meta-analysis were mainly conducted in Europe, the United States and Oceania, and studies on Asian populations were lacking. Therefore, the results may not be generalized to other populations with different baseline risks for melanoma. The ability to correct for confounders is limited, although we performed subgroup analyses for geographic areas, sun exposure factors, and so on. In addition, our subgroup analysis suggests that daylight exposure factors may contribute to changes in the intensity of the association; (5) potential publication bias may influence the research results, but as shown in the funnel plot, little evidence of publication bias was found in this meta-analysis.

The association between melanoma risk and hormonal/reproductive factors requires further investigation. In vitro, sex hormones stimulate melanogenesis with direct action on the melanocytes. Sex hormones such as estrogen act through their cognate receptors: Estrogen Receptor alfa $(\mathrm{ER} \alpha)$ and Estrogen Receptor beta $(\mathrm{ER} \beta) . \mathrm{ER} \alpha$ is widely accepted to play a role in tumorigenesis by stimulating cell proliferation, while ER $\beta$ seem to have a significant antitumor activity. ${ }^{62}$ Recent studies concluded that estrogen signaling depends primarily on the balance between expression of ER $\alpha$ and ER $\beta$. Depending on the predominant estrogen receptor, the overall effect in a specific tissue will be to stimulate cell growth and proliferation, or conversely, to inhibit their growth. ${ }^{63}$ Another estrogen receptor, G proteincoupled estrogen receptor (GPER), which is an integral membrane protein expressed by melanoma cells, has been recently reported to mediate both tumor-promoting and antitumor effects. GPER agonists inhibit the proliferation of melanoma cells in vitro. ${ }^{64}$ However, the use of estrogen-receptor modulators in the treatment of melanoma still remains controversial, such as the tamoxifen (TAM). The antitumor and pro-survival effects of tamoxifen may depend on the different ER $\alpha / E R \beta$ ratios in a given tissue. ${ }^{65,66}$ Therefore, further studies are required and may reveal new pathophysiological mechanisms and new therapeutic targets for cutaneous melanoma in the future.

\section{Conclusion}

In conclusion, the results of this meta-analysis suggest that long-term use of OC may increase the risk of melanoma in women, and that an incubation period of 15 to 19 years may be required to increase the risk of melanoma. However, the age at first use of OC and years since last use of $\mathrm{OC}$ is not associated with melanoma. HRT use is associated with an increased incidence of melanoma in women, especially with an increased risk of SSM, and estrogen and estradiol may be the main active agents that contribute to the increased risk of melanoma, but these results may be due to a combination of sun exposure factors. Decreased number of parity and age $\geq 20$ years at the first birth were associated with an increased risk of melanoma in women, while menopausal status, and age at menarche were not associated with the incidence of melanoma in women. However, the results may be contingent or due to different active ingredients acting on different signaling pathways. Therefore, further large-scale prospective studies are necessary to reveal new pathophysiological mechanisms and new therapeutic targets for cutaneous melanoma.

\section{Funding}

This study was supported by the Science and Technology Development plan of Jilin Province, No. 20190701061GH, No. 20200601010JC and No. 20200201598JC.

\section{Disclosure}

The authors report no conflicts of interest in this work. 


\section{References}

1. Rastrelli M, Tropea S, Rossi CR, Alaibac M. Melanoma: epidemiology, risk factors, pathogenesis, diagnosis and classification. In Vivo (Brooklyn). 2014;28(6):1005-1011.

2. Fattouh K, Ducroux E, Decullier E, et al. Increasing incidence of melanoma after solid organ transplantation: a retrospective epidemiological study. Transpl Int. 2017;30(11):1172-1180. doi:10.1111/ tri. 13011

3. Rizvi SMH, Aagnes B, Holdaas H, et al. Long-term change in the risk of skin cancer after organ transplantation: a population-based nationwide cohort study. JAMA Dermatol. 2017;153(12):1270-1277. doi:10.1001/jamadermatol.2017.2984

4. Carr S, Smith C, Wernberg J. Epidemiology and risk factors of melanoma. Surg Clin North Am. 2020;100(1):1-12. doi:10.1016/j. suc.2019.09.005

5. Ascha M, Ascha MS, Tanenbaum J, Bordeaux JS. Risk factors for melanoma in renal transplant recipients. JAMA Dermatol. 2017;153 (11):1130-1136. doi:10.1001/jamadermatol.2017.2291

6. Robsahm TE, Bergva G, Hestvik UE, Moller B. Sex differences in rising trends of cutaneous malignant melanoma in Norway, 19542008. Melanoma Res. 2013;23(1):70-78. doi:10.1097/ CMR.0b013e328

$35 \mathrm{c} 7 \mathrm{e} 48$

7. Reed KB, Brewer JD, Lohse CM, Bringe KE, Pruitt CN, Gibson LE. Increasing incidence of melanoma among young adults: an epidemiological study in Olmsted County, Minnesota. Mayo Clin Proc. 2012;87(4):328-334. doi:10.1016/j.mayocp.2012.01.010

8. Enninga EAL, Moser JC, Weaver AL, et al. Survival of cutaneous melanoma based on sex, age, and stage in the United States, 19922011. Cancer Med. 2017;6(10):2203-2212. doi:10.1002/ cam4.1152

9. Van Rooij N, Adams A, De'Ambrosis B, Nathan V, Hayward N, Whiteman D. Cluster of pregnancy-associated melanoma: a case report and brief update. $J$ Dermatol. 2020;47:1054-1057. doi:10.1111/1346-8138.15455

10. Anaya-Prado R, Ruvalcaba-Soto EN, Anaya-Fernandez MM, et al. Melanoma and pregnancy. A case report. J Obstet Gynaecol. 2017;37 (7):950-951. doi:10.1080/01443615.2017.1308320

11. Wielowieyska-Szybinska DK, Spalkowska M, Wojas-Pelc A. Melanoma in pregnancy: a case report and review of the literature. Postepy Dermatol Alergol. 2015;32(6):483-487. doi:10.5114/ pdia.2014.44006

12. Zampetti A, Feliciani C, Landi F, Capaldo ML, Rotoli M, Amerio PL. Management and dermoscopy of fast-growing nevi in pregnancy: case report and literature review. J Cutan Med Surg. 2006;10 (5):249-252. doi:10.2310/7750.2006.00047

13. Feskanich D, Hunter DJ, Willett WC, et al. Oral contraceptive use and risk of melanoma in premenopausal women. $\mathrm{Br} J$ Cancer. 1999;81(5):918-923. doi:10.1038/sj.bjc.6690787

14. Koomen ER, Joosse A, Herings RM, Casparie MK, Guchelaar HJ, Nijsten T. Estrogens, oral contraceptives and hormonal replacement therapy increase the incidence of cutaneous melanoma: a populationbased case-control study. Ann Oncol. 2009;20(2):358-364. doi:10.1093/annonc/mdn589

15. Hicks BM, Kristensen KB, Pedersen SA, Holmich LR, Pottegard A. Hormone replacement therapy and the risk of melanoma in postmenopausal women. Hum Reprod. 2019;34(12):2418-2429. doi:10.1093/humrep/dez222

16. Osterlind A, Tucker MA, Stone BJ, Jensen OM. The Danish casecontrol study of cutaneous malignant melanoma. III. Hormonal and reproductive factors in women. Int J Cancer. 1988;42(6):821-824. doi:10.1002/ijc.2910420603

17. Beral V, Ramcharan S, Faris R. Malignant melanoma and oral contraceptive use among women in California. Br J Cancer. 1977;36 (6):804-809. doi:10.1038/bjc.1977.265
18. Adam SA, Sheaves JK, Wright NH, Mosser G, Harris RW, Vessey MP. A case-control study of the possible association between oral contraceptives and malignant melanoma. Br J Cancer. 1981;44 (1):45-50. doi:10.1038/bjc. 1981.145

19. Bain C, Hennekens CH, Speizer FE, Rosner B, Willett W, Belanger C. Oral contraceptive use and malignant melanoma. $J$ Natl Cancer Inst. 1982;68(4):537-539.

20. Beral V, Evans S, Shaw H, Milton G. Oral contraceptive use and malignant melanoma in Australia. Br J Cancer. 1984;50(5):681-685. doi:10.1038/bjc. 1984.236

21. Holman CD, Armstrong BK, Heenan PJ. Cutaneous malignant melanoma in women: exogenous sex hormones and reproductive factors. Br J Cancer. 1984;50(5):673-680. doi:10.1038/bjc.1984.235

22. Helmrich SP, Rosenberg L, Kaufman DW, et al. Lack of an elevated risk of malignant melanoma in relation to oral contraceptive use. $J$ Natl Cancer Inst. 1984;72(3):617-620.

23. Zanetti R, Franceschi S, Rosso S, Bidoli E, Colonna S. Cutaneous malignant melanoma in females: the role of hormonal and reproductive factors. Int J Epidemiol. 1990;19(3):522-526. doi:10.1093/ije/19.3.522

24. Le MG, Cabanes PA, Desvignes V, Chanteau MF, Mlika N, Avril MF. Oral contraceptive use and risk of cutaneous malignant melanoma in a case-control study of French women. Cancer Causes Control. 1992;3(3):199-205. doi:10.1007/BF00124252

25. Palmer JR, Rosenberg L, Strom BL, et al. Oral contraceptive use and risk of cutaneous malignant melanoma. Cancer Causes Control. 1992;3(6):547-554. doi:10.1007/BF00052752

26. Holly EA, Cress RD, Ahn DK. Cutaneous melanoma in women. III. Reproductive factors and oral contraceptive use. Am J Epidemiol. 1995;141(10):943-950. doi:10.1093/oxfordjournals.aje.a117361

27. Smith MA, Fine JA, Barnhill RL, Berwick M. Hormonal and reproductive influences and risk of melanoma in women. Int J Epidemiol. 1998;27(5):751-757. doi:10.1093/ije/27.5.751

28. Westerdahl J, Olsson H, Masback A, Ingvar C, Jonsson N. Risk of malignant melanoma in relation to drug intake, alcohol, smoking and hormonal factors. Br J Cancer. 1996;73(9):1126-1131. doi:10.1038/ bjc. 1996.216

29. Freedman DM, Sigurdson A, Doody MM, Rao RS, Linet MS. Risk of melanoma in relation to smoking, alcohol intake, and other factors in a large occupational cohort. Cancer Causes Control. 2003;14(9):847857. doi:10.1023/B:CACO.0000003839.56954.73

30. Naldi L, Altieri A, Imberti GL, et al. Cutaneous malignant melanoma in women. Phenotypic characteristics, sun exposure, and hormonal factors: a case-control study from Italy. Ann Epidemiol. 2005;15 (7):545-550. doi:10.1016/j.annepidem.2004.10.005

31. Lea CS, Holly EA, Hartge P, et al. Reproductive risk factors for cutaneous melanoma in women: a case-control study. $\mathrm{Am} J$ Epidemiol. 2007;165(5):505-513. doi:10.1093/aje/kwk040

32. Holly EA, Weiss NS, Liff JM. Cutaneous melanoma in relation to exogenous hormones and reproductive factors. $J$ Natl Cancer Inst. 1983;70(5):827-831.

33. Adami HO, Persson I, Hoover R, Schairer C, Bergkvist L. Risk of cancer in women receiving hormone replacement therapy. Int $J$ Cancer. 1989;44(5):833-839. doi:10.1002/ijc.2910440515

34. Holly EA, Cress RD, Ahn DK. Cutaneous melanoma in women: ovulatory life, menopause, and use of exogenous estrogens. Cancer Epidemiol Biomarkers Prev. 1994;3(8):661-668.

35. Cervenka I, Mahamat-Saleh Y, Savoye I, et al. Oral contraceptive use and cutaneous melanoma risk: a French prospective cohort study. Int J Cancer. 2018;143(10):2390-2399. doi:10.1002/ijc.31644

36. Donley GM, Liu WT, Pfeiffer RM, et al. Reproductive factors, exogenous hormone use and incidence of melanoma among women in the United States. Br J Cancer. 2019;120(7):754-760. doi:10.1038/ s41416-019-0411-z

37. Cervenka I, Al Rahmoun M, Mahamat-Saleh Y, et al. Postmenopausal hormone use and cutaneous melanoma risk: a French prospective cohort study. Int J Cancer. 2019;145(7):1754-1767. doi:10.1002/ijc.32150 
38. Cervenka I, Al Rahmoun M, Mahamat-Saleh Y, et al. Exogenous hormone use and cutaneous melanoma risk in women: the European prospective investigation into cancer and nutrition. Int $J$ Cancer. 2020;146(12):3267-3280. doi:10.1002/ijc.32674

39. Zaridze D, Mukeria A, Duffy S. Risk factors for skin melanoma in Moscow. Int J Cancer. 1992;52(1):159-161. doi:10.1002/ijc.29105 20128

40. De Giorgi V, Gori A, Savarese I, et al. Role of BMI and hormone therapy in melanoma risk: a case-control study. J Cancer Res Clin Oncol. 2017;143(7):1191-1197. doi:10.1007/s00432-0172387-5

41. Lambe M, Thorn M, Sparen P, Bergstrom R, Adami HO. Malignant melanoma: reduced risk associated with early childbearing and multiparity. Melanoma Res. 1996;6(2):147-153. doi:10.1097/00008390199604000-00010

42. Neale RE, Darlington S, Murphy MF, Silcocks PB, Purdie DM, Talback M. The effects of twins, parity and age at first birth on cancer risk in Swedish women. Twin Res Hum Genet. 2005;8 (2):156-162. doi:10.1375/twin.8.2.156

43. Stoer NC, Botteri E, Ghiasvand R, et al. Reproductive factors and risk of melanoma: a population-based cohort study. $\mathrm{Br} J$ Dermatol. 2019;181(2):282-289. doi:10.1111/bjd.17771

44. Liberati A, Altman DG, Tetzlaff J, et al. The PRISMA statement for reporting systematic reviews and meta-analyses of studies that evaluate healthcare interventions: explanation and elaboration. BMJ. 2009;339:b2700. doi:10.1136/bmj.b2700

45. GA SB W, O'Connell D, et al. The Newcastle-Ottawa Scale (NOS) for Assessing the Quality of Nonrandomised Studies in MetaAnalyses. Ottawa: Ottawa Health Research Institute University of Ottawa; 2000.

46. Davies HT, Crombie IK, Tavakoli M. When can odds ratios mislead? BMJ. 1998;316(7136):989-991. doi:10.1136/bmj.316.7136.989

47. DerSimonian R, Laird N. Meta-analysis in clinical trials. Control Clin Trials. 1986;7(3):177-188. doi:10.1016/0197-2456(86)90046-2

48. Higgins JPGS. Cochrane handbook for systematic reviews of interventions version 5.1.0. Cochrane Collaboration. 2011

49. Begg CB, Mazumdar M. Operating characteristics of a rank correlation test for publication bias. Biometrics. 1994;50(4):1088-1101. doi: $10.2307 / 2533446$

50. Egger M, Davey Smith G, Schneider M, Minder C. Bias in metaanalysis detected by a simple, graphical test. BMJ. 1997;315 (7109):629-634. doi:10.1136/bmj.315.7109.629

51. Young P, Purdie D, Jackman L, Molloy D, Green A. A study of infertility treatment and melanoma. Melanoma Res. 2001;11(5):535541. doi:10.1097/00008390-200110000-00015

52. Vessey M, Painter R. Oral contraceptive use and cancer. Findings in a large cohort study, 19682004. Br J Cancer. 2006;95(3):385-389. doi:10.1038/sj.bjc. 6603260

53. Hannaford PC, Selvaraj S, Elliott AM, Angus V, Iversen L, Lee AJ. Cancer risk among users of oral contraceptives: cohort data from the Royal College of General Practitioner's oral contraception study. BMJ. 2007;335(7621):651. doi:10.1136/bmj.39289.649 410.55
54. Hannibal CG, Jensen A, Sharif H, Kjaer SK. Malignant melanoma risk after exposure to fertility drugs: results from a large Danish cohort study. Cancer Causes Control. 2008;19(7):759-765. doi:10.1007/s10552-008-9138-5

55. Kvaskoff M, Bijon A, Mesrine S, Boutron-Ruault MC, ClavelChapelon F. Cutaneous melanoma and endogenous hormonal factors: a large French prospective study. Am J Epidemiol. 2011;173 (10):1192-1202. doi:10.1093/aje/kwq503

56. Botteri E, Stoer NC, Sakshaug S, et al. Menopausal hormone therapy and risk of melanoma: do estrogens and progestins have a different role? Int J Cancer. 2017;141(9):1763-1770. doi:10.1002/ijc.30878

57. Mueller K, Verzi AE, Bhatt K, et al. Melanoma and chronic exposure to contraceptives containing microdoses of ethinylestradiol in young women: a retrospective study from the Research on Adverse Drug Events and Reports (RADAR) project comprising a large Midwestern U.S. patient population. J Eur Acad Dermatol Venereol. 2018;32(3): e87e88. doi:10.1111/jdv.14534

58. Pfahlberg A, Hassan K, Wille L, Lausen B, Gefeller O. Systematic review of case-control studies: oral contraceptives show no effect on melanoma risk. Public Health Rev. 1997;25(34):309-315.

59. Karagas MR, Stukel TA, Dykes J, et al. A pooled analysis of 10 casecontrol studies of melanoma and oral contraceptive use. Br J Cancer. 2002;86(7):1085-1092. doi:10.1038/sj.bjc.6600196

60. Gefeller O, Hassan K, Wille L. Cutaneous malignant melanoma in women and the role of oral contraceptives. Br J Dermatol. 1998;138 (1):122-124. doi:10.1046/j.1365-2133.1998.02037.x

61. Gandini S, Iodice S, Koomen E, Di Pietro A, Sera F, Caini S. Hormonal and reproductive factors in relation to melanoma in women: current review and meta-analysis. Eur J Cancer. 2011;47 (17):2607-2617. doi:10.1016/j.ejca.2011.04.023

62. Barone I, Brusco L, Fuqua SA. Estrogen receptor mutations and changes in downstream gene expression and signaling. Clin Cancer Res. 2010;16(10):2702-2708. doi:10.1158/1078-0432.CCR-09-1753

63. Caruntu C, Mirica A, Rosca AE, et al. The role of estrogens and estrogen receptors in melanoma development and progression. Acta Endocrinol. 2016;12(2):234-241. doi:10.4183/aeb.2016.234

64. Ribeiro MPC, Santos AE, Custodio JBA. The activation of the G protein-coupled estrogen receptor (GPER) inhibits the proliferation of mouse melanoma K1735-M2 cells. Chem Biol Interact. 2017;277:176-184. doi:10.1016/j.cbi.2017.09.017

65. Lens MB, Reiman T, Husain AF. Use of tamoxifen in the treatment of malignant melanoma. Cancer. 2003;98(7):1355-1361. doi:10.1002/ cncr. 11644

66. Beguerie JR, Xingzhong J, Valdez RP. Tamoxifen vs. non-tamoxifen treatment for advanced melanoma: a meta-analysis. Int J Dermatol. 2010;49(10):1194-1202. doi:10.1111/j.1365-4632.2010.04529.x
Clinical Epidemiology

\section{Publish your work in this journal}

Clinical Epidemiology is an international, peer-reviewed, open access, online journal focusing on disease and drug epidemiology, identification of risk factors and screening procedures to develop optimal preventative initiatives and programs. Specific topics include: diagnosis, prognosis, treatment, screening, prevention, risk factor modification,

Submit your manuscript here: https://www.dovepress.com/clinical-epidemiology-journal systematic reviews, risk \& safety of medical interventions, epidemiology \& biostatistical methods, and evaluation of guidelines, translational medicine, health policies \& economic evaluations. The manuscript management system is completely online and includes a very quick and fair peer-review system, which is all easy to use. 